\title{
Modularity of genes involved in local adaptation to climate despite physical linkage
}

\author{
Katie E Lotterhos ${ }^{1 *}$ D, Sam Yeaman², Jon Degner ${ }^{3}$, Sally Aitken ${ }^{3}$ and Kathryn A Hodgins ${ }^{4}$
}

\begin{abstract}
Background: Linkage among genes experiencing different selection pressures can make natural selection less efficient. Theory predicts that when local adaptation is driven by complex and non-covarying stresses, increased linkage is favored for alleles with similar pleiotropic effects, with increased recombination favored among alleles with contrasting pleiotropic effects. Here, we introduce a framework to test these predictions with a co-association network analysis, which clusters loci based on differing associations. We use this framework to study the genetic architecture of local adaptation to climate in lodgepole pine, Pinus contorta, based on associations with environments.

Results: We identify many clusters of candidate genes and SNPs associated with distinct environments, including aspects of aridity and freezing, and discover low recombination rates among some candidate genes in different clusters. Only a few genes contain SNPs with effects on more than one distinct aspect of climate. There is limited correspondence between co-association networks and gene regulatory networks. We further show how associations with environmental principal components can lead to misinterpretation. Finally, simulations illustrate both benefits and caveats of co-association networks.

Conclusions: Our results support the prediction that different selection pressures favor the evolution of distinct groups of genes, each associating with a different aspect of climate. But our results went against the prediction that loci experiencing different sources of selection would have high recombination among them. These results give new insight into evolutionary debates about the extent of modularity, pleiotropy, and linkage in the evolution of genetic architectures.
\end{abstract}

Keywords: Landscape genomics, Genome-environment associations, Genome-wide association studies (GWAS), Conifers, Linkage disequilibrium, Ion antiporters, Auxin biosynthesis, Flowering time

\section{Background}

Pleiotropy and linkage are fundamental aspects of genetic architecture [1]. Pleiotropy is when a gene has effects on multiple distinct traits. Pleiotropy may hinder the rate of adaptation by increasing the likelihood that genetic changes have a deleterious effect on at least one trait $[2$, 3]. Similarly, linkage among genes experiencing different kinds of selection can facilitate or hinder adaptation [4-6]. Despite progress in understanding the underlying pleiotropic nature of phenotypes and the influence of

\footnotetext{
*Correspondence: k.lotterhos@northeastern.edu

'Department of Marine and Environmental Sciences, Northeastern Marine

Science Center, 430 Nahant Rd, Nahant, MA 01908, USA

Full list of author information is available at the end of the article
}

pleiotropy on the rate of adaptation to specific conditions [7], we have an incomplete understanding of the extent and magnitude of linkage and pleiotropy in the local adaptation of natural populations to the landscapes and environments in which they are found.

Here, we aim to characterize the genetic architecture of adaptation to the environment, including the number of separate components of the environment in which a gene affects fitness (a form of "selectional pleiotropy," Table 1) [8]. Genetic architecture is an encompassing term used to describe the pattern of genetic features that build and control a trait, and includes statements about the number of genes or alleles involved, their arrangement on chromosomes, the distribution of their effects,

(c) The Author(s). 2018 Open Access This article is distributed under the terms of the Creative Commons Attribution 4.0 International License (http://creativecommons.org/licenses/by/4.0/), which permits unrestricted use, distribution, and 
Table 1 Overview of terminology used in the literature regarding pleiotropy and modularity

\begin{tabular}{|c|c|c|}
\hline Term & Meaning & References \\
\hline Genetic architecture & $\begin{array}{l}\text { Genetic architecture refers to the pattern of genetic effects that build and control a facet of the } \\
\text { organism (character, trait, or fitness). A description of genetic architecture includes statements about } \\
\text { gene and allele number, the distribution of allelic and mutation effects, patterns of pleiotropy, and } \\
\text { recombination rates among causal loci on chromosomes }\end{array}$ & [1] \\
\hline Selectional pleiotropy & $\begin{array}{l}\text { The number of separate components of fitness a mutation effects. Traits are defined by the action of } \\
\text { selection and not by the intrinsic attributes of the organism }\end{array}$ & [8] \\
\hline $\begin{array}{l}\text { Antagonistic pleiotropy at } \\
\text { a single allele }\end{array}$ & $\begin{array}{l}\text { In the context of this study, an allele exhibits antagonistic pleiotropy if it has different effects on fitness } \\
\text { at different extremes of an environmental variable (e.g., positive effects on fitness in cold environments } \\
\text { and negative effects in warm environments), which results in an association between the allele } \\
\text { frequency and the environmental variable }\end{array}$ & [9] \\
\hline Environmental pleiotropy & $\begin{array}{l}\text { Genes affect fitness in multiple distinct aspects of the multivariate environment, where each aspect is } \\
\text { defined by the action of selection }\end{array}$ & This study \\
\hline $\begin{array}{l}\text { Modularity or modular } \\
\text { genetic architecture }\end{array}$ & $\begin{array}{l}\text { A modular unit is a complex of elements (characters or genes) that (1) collectively serve a similar } \\
\text { functional role, (2) are tightly integrated by strong pleiotropic effects of genetic variation, and ( } 3 \text { ) are } \\
\text { relatively independent from other such units. Pleiotropic effects may be on traits or on fitness, and are } \\
\text { limited to elements within a module, with a suppression of pleiotropic effects between different } \\
\text { modules (Fig. 1a, left column). Genes within a module may or may not be physically linked }\end{array}$ & [25] \\
\hline $\begin{array}{l}\text { Co-association network } \\
\text { analysis }\end{array}$ & $\begin{array}{l}\text { An application of network theory used to identify modules of loci that are similar in their associations } \\
\text { across many variables }\end{array}$ & This study \\
\hline Co-association module & $\begin{array}{l}\text { A group of SNPs that show associations with a distinct selective environmental factor. These modules } \\
\text { can be thought of as "variational" modules (sensu [19]), which are composed of features that vary } \\
\text { together and are relatively independent of other such sets of features. In practice, co-association } \\
\text { modules are inferred by their similarity in associations with multiple variables }\end{array}$ & This study \\
\hline $\begin{array}{l}\text { Selective environmental } \\
\text { factors }\end{array}$ & $\begin{array}{l}\text { The specific aspect of the multivariate environment to which a SNP adapts on a geographic landscape. } \\
\text { In practice, these are inferred by the environmental variables that associate with candidate SNPs within } \\
\text { co-association modules }\end{array}$ & This study \\
\hline
\end{tabular}

and patterns of pleiotropy (Table 1). We can measure many parameters to characterize environments (e.g., temperature, latitude, precipitation), but the variables we define may not correspond to the environmental factors that matter for an organism's fitness. A major hurdle in understanding how environments shape fitness is defining the environment based on factors that drive selection and local adaptation and not by the intrinsic attributes of the organism or by the environmental variables we happen to measure.

In local adaptation to climate, an allele that has different effects on fitness at different extremes of an environmental variable (e.g., positive effects on fitness in cold environments and negative effects in warm environments, often called "antagonistic pleiotropy," Table 1 [9]) will evolve to produce a clinal relationship between the allele frequency and that environmental factor [10-15]. While associations between allele frequencies and environmental factors have been well characterized across many taxa [16], whether genes affect fitness in multiple distinct aspects of the environment, which we call "environmental pleiotropy" (e.g., has effects on fitness in both cold and dry environments, Table 1), has not been well characterized [17]. This is because of conceptual issues that arise from defining environments along the univariate axes that we measure. For example, "cold" and "dry" might be a single selective optimum ("cold-dry") to which a gene adapts [7], but these two axes are typically analyzed separately. Moreover, climate variables such as temperature and precipitation may be highly correlated across landscapes, and this correlation structure makes inferring pleiotropy from signals of selection to climate difficult. Indeed, in their study of climate adaptation in Arabidopsis, Hancock et al. [17] noticed that candidate loci showed signals of selection in multiple environmental variables, potentially indicating pleiotropic effects. However, they also found that a substantial proportion of this overlap was due to correlations among climate variables on the landscape, and as a result, they were unable to fully describe pleiotropic effects.

Because of the conceptual issues described above, certain aspects of the genetic architecture of adaptation to landscapes have not been well characterized, particularly the patterns of linkage among genes adapting to distinct environmental factors, and the degree of pleiotropic effects of genes on fitness in distinct environments. These aspects of genetic architecture are important to characterize, in order to test the theoretical predictions described below, and to inform the considerable debate about whether organisms have a modular organization of gene effects on phenotypes or fitness components, versus universal effects of genes on all phenotypes or fitness components (Fig. 1a, compare left to right column) [18-24].

Modular genetic architectures are characterized by extensive pleiotropic effects among elements within a module, and a suppression of pleiotropic effects between 


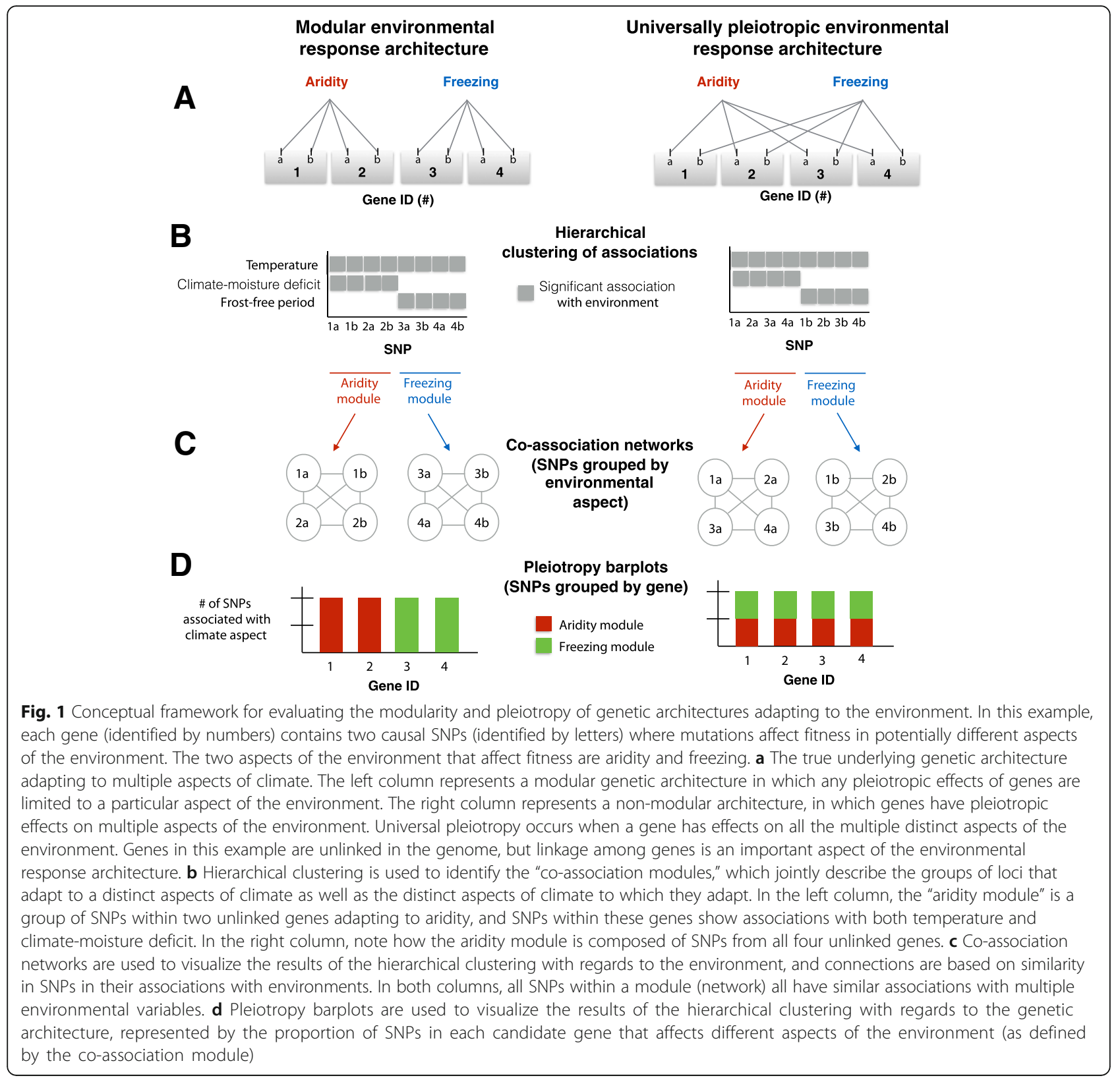

different modules [25]. Note that modularity in this study refers to similarity in the effects of loci on fitness and not necessarily to the physical location of loci on chromosomes or to participation in the same gene regulatory network. Theory predicts that modular genetic architectures will be favored when genomes face complex spatial and temporal environments [26] or when multiple traits are under a combination of directional and stabilizing selection (because modularity allows adaptation to take place in one trait without undoing the adaptation achieved by another trait) [25, 27]. Adaptation to climate on a landscape fits these criteria because environmental variation among populations is complex-with multiple abiotic and biotic challenges occurring at different spatial scales-and traits are thought to be under stabilizing selection within populations but directional selection among populations [28].

Clusters of physically linked loci subject to the same selective environment, as well as a lack of physical linkage among loci subject to different selection pressures, are expected based on theory. When mutations are subject to the same selection pressure, recombination can bring variants with similar effects together and allow evolution to proceed faster [29]. Clusters of adaptive loci can also arise through genomic rearrangements that bring existing mutations together [30] or because new causal mutations linked to adaptive alleles have an increased establishment probability [31]. Similarly, clusters 
of locally adaptive loci are expected to evolve in regions of low recombination, such as inversions, because of the reduced gene flow these regions experience [32, 33]. In general, these linked clusters of adaptive loci are favored over evolutionary time because low recombination rates increase the rate at which they are inherited together. Conversely, selection will also act to disfavor linkage and increase recombination rates between genes adapting to different selection pressures [34-36]. Thus, genes adapting to different selection pressures would be unlikely to be physically linked or to have low recombination rates between them. In practice, issues can arise in inference because physical linkage will cause correlated responses to selection in neutral loci flanking a causal locus. Large regions of the genome can share similar patterns of association to a given environmental factor, such that many loci within a given candidate region are probably not causally responding to selection. Conversely, if linked genes are associated with completely different aspects of the selective environment, this is unlikely to arise by chance.

In summary, current analytical techniques have given limited insight into the genetic architectures of adaptation to environmental variation across natural landscapes. Characterizing the different aspects of the environment that act on genomes is difficult because measured variables are univariate and may not be representative of selection from the perspective of the organism and because of spatial correlations among environmental variables. Even when many variables are summarized with ordination such as principal components, the axes that explain the most variation in physical environment do not necessarily correspond to the axes that cause selection because the components are orthogonal [37]. Furthermore, the statistical methods widely used for inferring adaptation to climate are also univariate in the sense that they test for significant correlations between the frequency of a single allele and a single environmental variable (e.g., [38, 39, 40]). While some multivariate regression methods like redundancy analysis have been used to understand how multiple environmental factors shape genetic structure [41, 42], they still rely on ordination and have not been used to identify distinct evolutionary modules of loci.

Here, we aim to fill this gap by presenting a framework for characterizing the genetic architecture of adaptation to the environment, through the joint inference of modules of loci that associate with distinct environmental factors that we call "co-association modules" (Table 1, Fig. 1), as well as the distinct factors of the environment to which they associate. Using this framework, we can characterize some aspects of genetic architecture, including modularity and linkage, that have not been well studied in the adaptation of genomes to environments.
We tested the hypotheses that (i) the genetic architecture of adaptation to complex environments is modular and (ii) that loci in different modules have evolved over time to be unlinked in the genome.

The framework is illustrated in Fig. 1 for four hypothetical genes adapted to two distinct aspects of climate (freezing and aridity). In this figure, we compare the patterns expected for (i) a modular architecture (left column, where pleiotropic fitness effects of a gene are limited to one particular climatic factor) to (ii) a highly environmentally pleiotropic architecture (right column, where genes have pleiotropic effects on adaptation to distinct climatic factors). Candidate SNPs are first identified by the significance of the univariate associations between allele frequency and the measured environmental variables, evaluated against what would be expected by neutrality. Then, hierarchical clustering of candidate SNP allele associations with environments is used to identify co-association modules (Fig. 1b) [43-45]. These modules can be visualized with a co-association network analysis, which identifies groups of loci that may covary with one environmental variable but covary in different ways with another, revealing patterns that are not evident through univariate analysis (Fig. 1c). By defining the distinct aspects of the selectional environment (Table 1) for each module through their environmental associations, we can infer pleiotropic effects of genes through the associations their SNPs have with distinct selective environmental factors (Fig. 1d). In this approach, the genetic effects of loci on different traits under selection are unknown, and we assume that each aspect of the multivariate environment selects for a trait or suite of traits that can be inferred by connecting candidate loci directly to the environmental factors that select for particular allelic combinations.

We apply this new approach to characterize the genetic architecture of local adaptation to climate in lodgepole pine (Pinus contorta) using a previously published exome capture dataset [46-48] from trees that inhabit a wide range of environments across their range, including freezing temperatures, precipitation, and aridity [49-52]. Lodgepole pine is a coniferous species inhabiting a wide range of environments in northwestern North America and exhibits isolation by distance population structure across the range [46]. Previous work based on reciprocal transplants and common garden experiments has shown extensive local adaptation [46, 53, 54]. We recently used this dataset to study convergent adaptation to freezing between lodgepole pine and the interior spruce complex (Picea glauca x Picea engelmannii) [46-48]. However, the comparative approach was limited to discovering parallel patterns between species and did not examine selective factors unique to one species. As in most other systems, the genomic architecture in pine underlying 
local adaptation to the multivariate environment has not been well characterized, and our reanalysis yields several new biological insights overlooked by the comparative approach.

We assessed the benefits and caveats of this new framework by comparing it with other multivariate approaches (based on principal components) and by evaluating it with simulated data. The evaluation with simulations yielded several important insights, including the importance of using strict criteria to exclude loci with false positive associations with environments. Thus, a key starting point for inferring co-association modules is a good set of candidate SNPs for adaptation. We developed this candidate set by first identifying top candidate genes for local adaptation (from a previously published set of genes that contained more outliers for genotype-environment associations and genotype-phenotype associations than expected by chance, [46]). We then identified "top candidate" SNPs within these top candidate genes as those whose allele frequencies were associated with at least one environmental variable above that expected by neutrality (using a criterion that excluded false positives in the simulated data described below). To this set of top candidate SNPs, we applied the framework outlined in Fig. 1 to characterize environmental modularity and linkage of the genetic architecture. The power of our dataset comes from including a large number of populations inhabiting diverse environments (>250), the accurate characterization of climate for each individual with 22 environmental variables, a high-quality exome capture dataset representing more than 500,000 single-nucleotide polymorphisms (SNPs) in $\sim 29,000$ genes [46-48], a mapping population that allows us to study recombination rates among genes, and an outgroup species that allowed us to determine the derived allele for most candidate SNPs. When such data is available, we find that this framework is useful for characterizing the environmental modularity and linkage relationships among candidate genes for local adaptation to multivariate environments.

\section{Results}

\section{Top candidate genes and top candidates SNPs}

The study of environmental pleiotropy and modularity is relevant only to loci under selection. Our "top candidate" approach identified a total of 108 top candidate genes out of a total of 29,920 genes. These contigs contained 801 top candidate SNPs (out of 585,270 exome SNPs) that were strongly associated with at least one environmental variable and were likely either causal or tightly linked to a causal locus. This set of top candidate SNPs was enriched for $X^{T} X$ outliers (Additional file 1: Figure $\mathrm{S} 1 ; X^{T} X$ is an analog of $F_{S T}$ that measures differentiation in allele frequencies across populations). To elucidate patterns of multivariate association, we applied the framework described in Fig. 1 to these 801 top candidate SNPs.

\section{Co-association modules}

Hierarchical clustering and co-association network analysis of top candidate SNPs revealed a large number of co-association modules, each of which contained SNPs from one or more genes. Each co-association module is represented by one or more top candidate SNPs (represented by nodes) that are connected by edges. The edges are drawn between two SNPs if they have similar associations with the environment below a distance threshold. The distance threshold was determined by simulation as a number that enriched connections among selected loci adapting to the same environmental variable and also decreased the number of connections to false positive loci (see the Results section "Simulated datasets").

For the purposes of illustration, we classified SNPs into four main groups, each with several co-association modules, according to the kinds of environmental variables they were most strongly associated with: Aridity, Freezing, Geography, and an assorted group we bin as "Multi" (Fig. 2a, b). Note that while we could have chosen a different number of groups, this would not have changed the underlying clustering of the SNPs revealed by co-association networks that are relevant to modularity (Fig. 2b-f). This division of data into groups was necessary to produce coherent visual network plots and to make data analyses more computationally efficient (we found when there were more than $\sim 20,000$ edges in the data, computation and plotting of the network were not feasible with the package). Note that SNPs in different groups are more dissimilar to SNPs in other groups than to those in the same group (based on the threshold we used to determine edges) and would not be connected by edges in a co-association module. Interestingly, this clustering by association signatures does not closely parallel the correlation structure among environmental variables themselves. For example, continentality (TD), degree days below $0{ }^{\circ} \mathrm{C}$ (DD_0), and latitude (LAT) are all relatively strongly correlated $(>0.5)$, while the "Freezing" SNPs are associated with continentality and degree days below 0 , but not latitude (Fig. 2a, b).

The co-association modules are shown in Fig. $2 \mathrm{c}-\mathrm{f}$. Each connected network of SNPs can be considered a group of loci that shows associations with a distinct environmental factor. The "Multi" group stands for multiple environments because these SNPs showed associations with 19 to 21 of the 22 environmental variables. This group consisted of 60 top candidate SNPs across just three genes, and undirected graph networks revealed two co-association modules within this group (Fig. 2c, Additional file 1: Figure S2). The "Aridity" group consisted of 282 SNPs across 28 genes and 


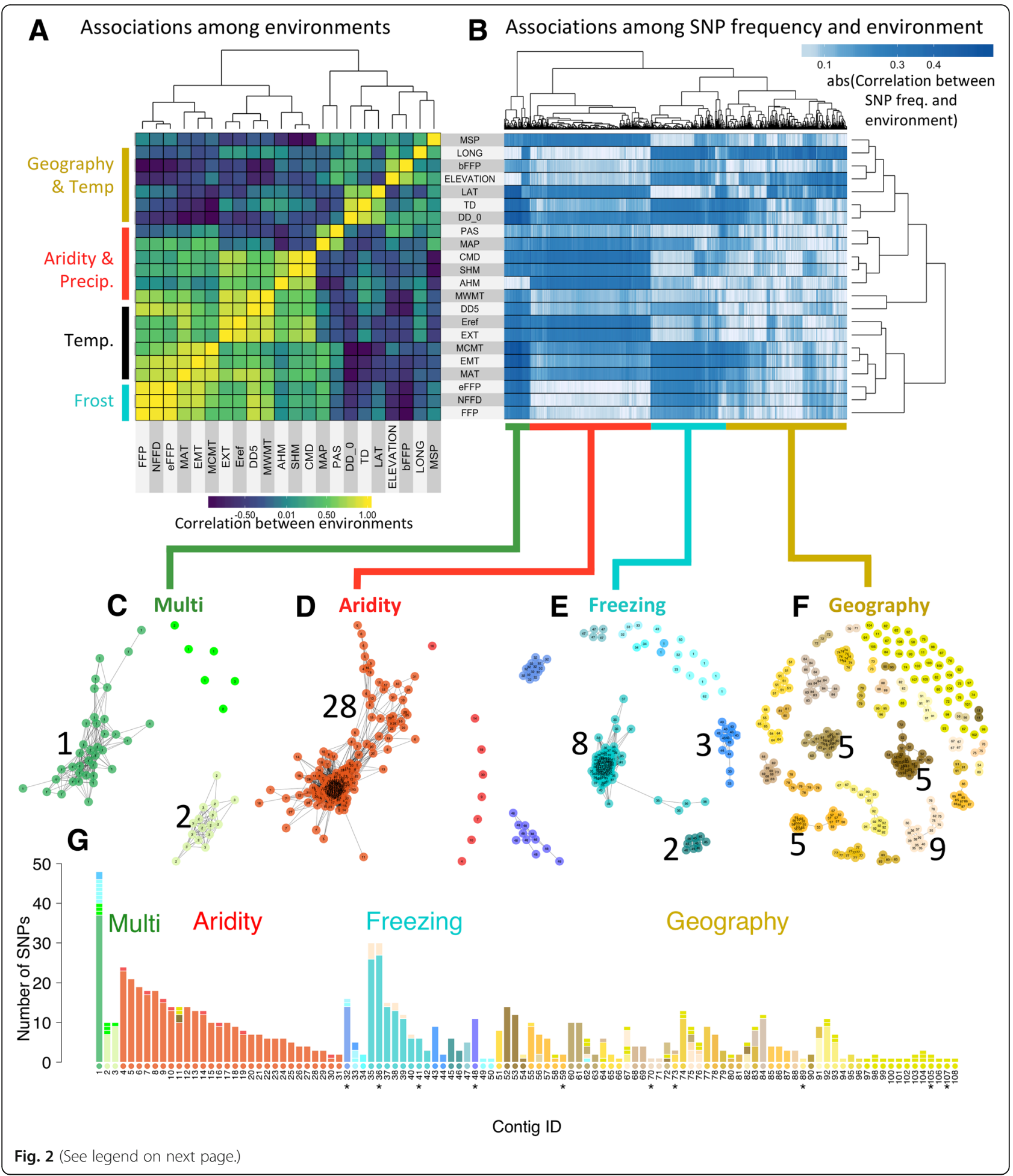


(See figure on previous page.)

Fig. 2 Co-association modules for Pinus contorta. a Correlations among environments measured by Spearman's $\rho$ plotted according to hierarchical clustering of environments. Abbreviations of the environmental variables can be found in Table 2. Note the general categories on the left side of the heatmap. b Hierarchical clustering of the absolute value of associations between allele frequencies (of SNPs in columns) and environments (in rows) measured by Spearman's $\mathbf{\rho}$. c-f Each co-association network represents a distinct co-association module, with color schemes according to the four major groups in the data. Each node is a SNP and is labeled with a number according to its exome contig, and a color according to its module - with the exceptions that modules containing a single SNP all give the same color within a major group. Numbers next to each module indicate the number of distinct genes involved (with the exception of the Geography group, where only modules with five or more genes are labeled). $\mathbf{g}$ The pleiotropy barplot, where each bar corresponds to a gene, and the colors represent the proportion of SNPs in each co-association module. Note that gene IDs are ordered by their co-association module, and the color of contig-IDs along the $x$ axis is determined by the co-association module that the majority of SNPs in that contig cluster with. Contigs previously identified as undergoing convergent evolution with spruce by Yeaman et al. [46] are indicated with an asterisk. Abbreviations: Temp, temperature; Precip, precipitation; freq, frequency

showed associations with climate-moisture deficit, annual heat:moisture index, mean summer precipitation, and temperature variables excluding those that were frost-related (Fig. 2b). All these SNPs were very similar in their patterns of association and grouped into a single co-association module (Fig. 2d, Additional file 1: Figure S3). The "Freezing" group consisted of 176 SNPs across 21 genes and showed associations with freezing variables including number of degree days below $0{ }^{\circ} \mathrm{C}$, mean coldest month temperature, and variables related to frost occurrence (Fig. 2b). SNPs from eight of the genes in this group formed a single module (gene no. 35-42), with the remaining SNPs mainly clustering by gene (Fig. 2e, Additional file 1: Figure S4). The final group, "Geography," consisted of 282 SNPs across 28 genes that showed consistent associations with the geographical variables elevation and longitude, but variable associations with other climate variables (Fig. 2b). This group consisted of several co-association modules containing one to nine genes (Fig. 2f, Additional file 1: Figure S5). Network analysis using population-structure-corrected associations between allele frequency and the environmental variables resulted in broadly similar patterns; although the magnitude of the correlations was reduced (Additional file 1: Figure S6, note

Table 2 Environmental variables measured for each sampling location, ordered by their abbreviations shown in Fig. 2a, b

\begin{tabular}{|c|c|c|}
\hline Abbreviation & Definition & Category \\
\hline MSP & May to September precipitation (mm) & Aridity \\
\hline LONG & Longitude & Geography \\
\hline bFPP & Day of the year frost-free period begins & Freezing \\
\hline ELEVATION & Elevation & Geography \\
\hline LAT & Latitude & Geography \\
\hline TD & Temperature difference (MWMT-MCMT) $\left({ }^{\circ} \mathrm{C}\right)$ & Freezing or Aridity \\
\hline DD_0 & Degree days below $0^{\circ} \mathrm{C}$ & Freezing \\
\hline PAS & Precipitation as snow (mm) & Aridity or Freezing \\
\hline MAP & Mean annual precipitation (mm) & Aridity \\
\hline CMD & Hargreaves climate-moisture deficit & Aridity \\
\hline SHM & Summer heat moisture index ((MWMT)/(MSP/1000)) & Aridity \\
\hline AHM & Annual heat moisture index (MAT+10)/(MAP/1000)) & Aridity \\
\hline MWMT & Mean warmest month temperature $\left({ }^{\circ} \mathrm{C}\right)$ & Aridity \\
\hline DD5 & Degree days above $5^{\circ} \mathrm{C}$ & Aridity \\
\hline Eref & Hargreaves reference evaporation & Aridity \\
\hline EXT & Extreme maximum temperature over 30 years $\left({ }^{\circ} \mathrm{C}\right)$ & Aridity \\
\hline MCMT & Mean coldest month temperature $\left({ }^{\circ} \mathrm{C}\right)$ & Freezing \\
\hline EMT & Extreme minimum temperature over 30 years $\left({ }^{\circ} \mathrm{C}\right)$ & Freezing \\
\hline MAT & Mean annual temperature $\left({ }^{\circ} \mathrm{C}\right)$ & Aridity or Freezing \\
\hline eFFP & Day of the year frost-free period ends & Freezing \\
\hline NFFD & Number of days without frost & Freezing \\
\hline FFP & Frost-free period (bFFP-eFFP) & Freezing \\
\hline
\end{tabular}


that neutral genetic structure was controlled for in choosing top candidates).

The pleiotropy barplot is visualized in Fig. $2 \mathrm{~g}$, where each gene is listed along the $x$ axis, the bar color indicates the co-association module, and the bar height indicates the number of SNPs clustering with that module. If each co-association module associates with a distinct aspect of the multivariate environment, then genes whose SNPs associate with different co-association modules (e.g., genes with different colors in their bars in Fig. 2g) might be considered to be environmentally pleiotropic. However, conceptual issues remain in inferring the extent of pleiotropy, because co-association modules within the Geography group, for instance, will be more similar to each other in their associations with environments than between a module in the Geography group and a module in the Multi group. For this reason, we are only inferring that our results are evidence of environmental pleiotropy when genes have SNPs in at least two of the four major groups in the data. For instance, gene no. 1, for which the majority of SNPs cluster with the Multi group, also has eight SNPs that cluster with the Freezing group (although they are not located in co-association modules with any genes defined by Freezing). In the Aridity group, gene no. 11 has three SNPs that also cluster with the Geography group (although they are not located in co-association modules with any genes defined by Geography). In the Freezing group, some genes located within the same co-association module (no. 35-40) also have SNPs that cluster with another module in the Geography group (with gene nos. 75-76; these are not physically linked to gene nos. 35-37, see below). Whether or not these are "true" instances of environmental pleiotropy remains to be determined by experiments. For the most part, however, the large majority of SNPs located within genes are in the same co-association module, or in modules located within one of the four main groups, so environmental pleiotropy at the gene level appears to be generally quite limited.

\section{Statistical and physical linkage disequilibrium}

To determine if grouping of SNPs into co-association modules corresponded to associations driven by statistical associations among genes measured by linkage disequilibrium (LD), we calculated mean LD among all SNPs in the top candidate genes (as the correlation in allele frequencies). We found that the co-association modules captured patterns of LD among the genes through their common associations with environmental variables (Additional file 1: Figure S7). There was higher than average LD within the co-association modules of the Multi, Aridity, and Freezing groups, and very low LD between the Aridity group and the other groups (Additional file 1: Figure S7). The LD among the other three groups (Multi, Freezing, and Geography) was small, but higher with each other than with Aridity. Thus, the co-association clustering corresponded to what we would expect based on LD among genes, with the important additional benefit of linking LD clusters to likely environmental drivers of selection.

The high LD observed within the four main environmental modules could arise via selection by the same factor of the multivariate environment, or via physical linkage on the chromosome, or both. We used a mapping population to disentangle these two hypotheses, by calculating recombination rates among the top candidate genes (see the Methods section "Recombination rates"). Of the 108 top candidate genes, 66 had SNPs that were represented in our mapping population. The recombination data revealed that all the genes in the Aridity group were in strong LD and physically linked (Fig. 3). Within the other three groups, we found physical proximity for only a few genes, typically within the same co-association module (but note that our mapping analysis does not have high power to infer recombination rate when loci are physically unlinked; see the "Methods" section). For example, a few co-association modules in the Geography group (comprised of gene nos. 53-54, no. 60-63, or no. 75-76) had very low recombination rates among them. Of the three genes forming the largest co-association module in the Freezing group that was represented in our mapping panel (no. 35-37), two were physically linked.

Strikingly, low recombination rates were estimated between some genes belonging to different co-association modules across the four main groups, even though there was little LD among SNPs in these genes (Fig. 3). This included a block of loci with low recombination comprised of genes from all four groups: eight genes from the Aridity co-association module, one gene from the large module in the Multi group, two genes from different co-association modules in the Freezing group, and seven genes from different co-association modules in the Geography group (upper diagonal of Fig. 3, see Additional file 1: Figure S8 for a reorganization of the recombination data and more intuitive visualization).

\section{Comparison to conclusions based on principal components of environments}

We compared the results from the co-association network analysis to associations with principal components (PC) of the environmental variables. Briefly, all environmental variables were input into a PC analysis, and associations between allele frequencies and PC axes were analyzed. We used the same criteria $\left(\log _{10} \mathrm{BF}>2\right.$ in Bayenv2) to determine if a locus was significant and compared (i) overlap with top candidate SNPs based on outliers from univariate associations with environments 


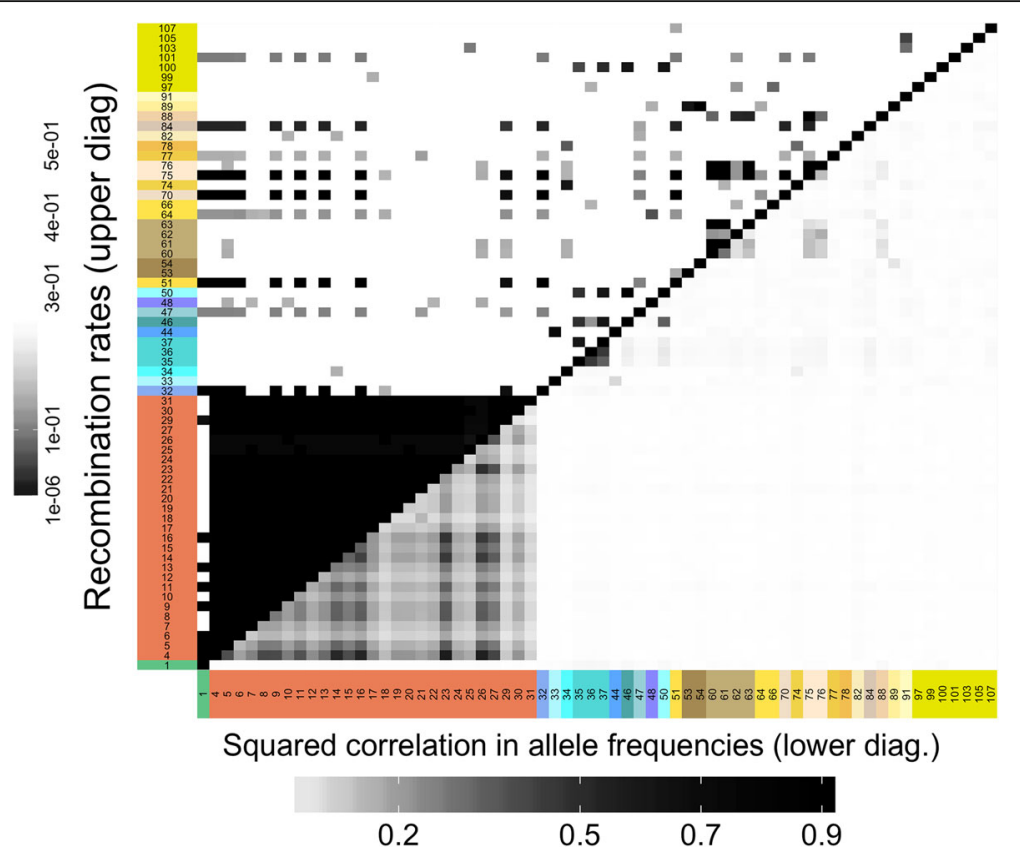

Fig. 3 Comparison of linkage disequilibrium (lower diagonal) and recombination rates (upper diagonal) for exome contigs. Only contigs with SNPs in the mapping panel are shown. Rows and column labels correspond to Fig. 2g. Darker areas represent either high physical linkage (low recombination) or high linkage disequilibrium (measured by the square of the correlation coefficient)

and (ii) interpretation of the selective environment based on loadings of environments to PC axes. The first three PC axes explained 44\% (PC1), 22\% (PC2), and 15\% (PC3) of the variance in environments (80\% total). Loadings of environment variables onto $\mathrm{PC}$ axes are shown in Additional file 1: Figure S9. A large proportion of top candidate SNPs in our study would not have been found if we had first done a PCA on the environments and then looked for outliers along PC axes: overall, $80 \%$ of the geography SNPs, $75 \%$ of the Freezing SNPs, $20 \%$ of the Aridity SNPs, and $10 \%$ of the Multi SNPs were not outliers along the first 10 PC axes and would have been missed.

Next, we evaluated whether interpretation of selective environments based on PCs was consistent with that based on associations with individual environmental factors. Some of the temperature and frost variables (MAT, mean annual temperature; EMT, extreme minimum temperature; DD0, degree days below $0{ }^{\circ} \mathrm{C}$; DD5, degree days above $5{ }^{\circ} \mathrm{C}$; bFFP, begin frost-free period; FFP, frost-free period; eFFP, end frost-free period; labels in Fig. 2a) had the highest loadings for PC1 (Additional file 1: Figure S9). Almost all of the SNPs in the Multi group (90\%) and 19\% of SNPs in the Freezing group were outliers along this axis (Additional file 1: Figure S10, note green outliers along $x$ axis from the Multi group; less than $2 \%$ of candidate SNPs in the other groups were outliers). For PC1, interpretation of the selective environment (e.g., MAT, DD0, FFP, eFFP, DD5) is partly consistent with the co-association network analysis. It was consistent because both Multi SNPs and Freezing SNPs show associations with all these variables (Fig. 2b). However, it was inconsistent because the Multi SNPs and Freezing SNPs had strong associations with other variables (e.g., Multi SNPs showed strong associations with latitude, and Freezing SNPs showed strong associations with longitude, Fig. 2b) that did not load strongly onto this axis, and so these putative environmental drivers would have been missed in an interpretation based on associations with principal components.

Many precipitation and aridity variables loaded strongly onto PC2, including mean annual precipitation, annual heat:moisture index, climate-moisture deficit, and precipitation as snow (Additional file 1: Figure S9). However, few top candidate SNPs were outliers along the PC2 axis: only 13\% of Freezing SNPs, $10 \%$ of Aridity SNPs, and less than 3\% of Multi or Geography SNPs were outliers (Additional file 1: Figure S10A, note lack of outliers on $y$ axis).

For PC3, latitude, elevation, and two frost variables (beginning frost-free period and frost-free period) had the highest loadings (Additional file 1: Figure S9). The majority $(78 \%)$ of the Aridity SNPs were outliers with PC3 (Additional file 1: Figure S10B, note outliers as orange dots on $y$ axis). Based on the PC association, this would lead one to conclude that the Aridity SNPs show associations with latitude, elevation, and frost-free period. While the Aridity SNPs do have strong associations with latitude 
(the fifth row in Fig. 2b), they show very weak associations with the beginning of frost-free period, elevation, and frost-free period length (the third, fourth, and last rows in Fig. 2b, respectively). Thus, interpretation of the environmental drivers of selection based on associations with PC3 would have been very different from the univariate associations.

\section{Interpretation of multivariate allele associations}

While the network visualization gave insight into patterns of LD among loci, it does not give insight into patterns of allele frequency change on the landscape, relative to the ancestral state. As illustrated above, principal components would not be useful for this latter visualization. Instead, we accomplished this by plotting the association of a derived allele with one environmental variable against the association of that allele with a second environmental variable. Note that when the two environmental variables themselves are correlated on the landscape, an allele with a larger association in one environment will also have a larger association with a second environment, regardless of whether or not selection is shaping those associations. We can visualize (i) the expected genome-wide covariance (given correlations between environmental variables; Fig. 2a) using shading of quadrants and (ii) the observed genome-wide covariance using a 95\% prediction ellipse (Fig. 4). Since alleles were coded according to their putative ancestral state in loblolly pine (Pinus taeda), the location of any particular SNP in the plot represents the bivariate environment in which the derived allele is found in higher frequency than the ancestral allele (Fig. 4). Visualizing the data in this way allows us to understand the underlying correlation structure of the data, as well as to develop testable hypotheses about the true selective environment and the fitness of the derived allele relative to the ancestral allele.

We overlaid the top candidate SNPs, colored according to their grouping in the co-association network analysis, on top of this genome-wide pattern (for the 668 of 801 top candidate SNPs for which the derived allele could be determined). We call these plots "galaxy biplots" because of the characteristic patterns we observed when visualizing data this way (Fig. 5). Galaxy biplots revealed that SNPs in the Aridity group showed associations with hot/dry versus cold/wet environments (red points in Fig. 5a), while SNPs in the Multi and Freezing groups showed patterns of associations with hot/wet versus cold/dry environments (blue and green dots in Fig. 5a). These outlier patterns became visually stronger for some SNPs and environments after correcting associations for population structure (compare Fig. 5a-b, structure-corrected allele frequencies calculated with Bayenv2, see the "Methods"). Most SNPs in

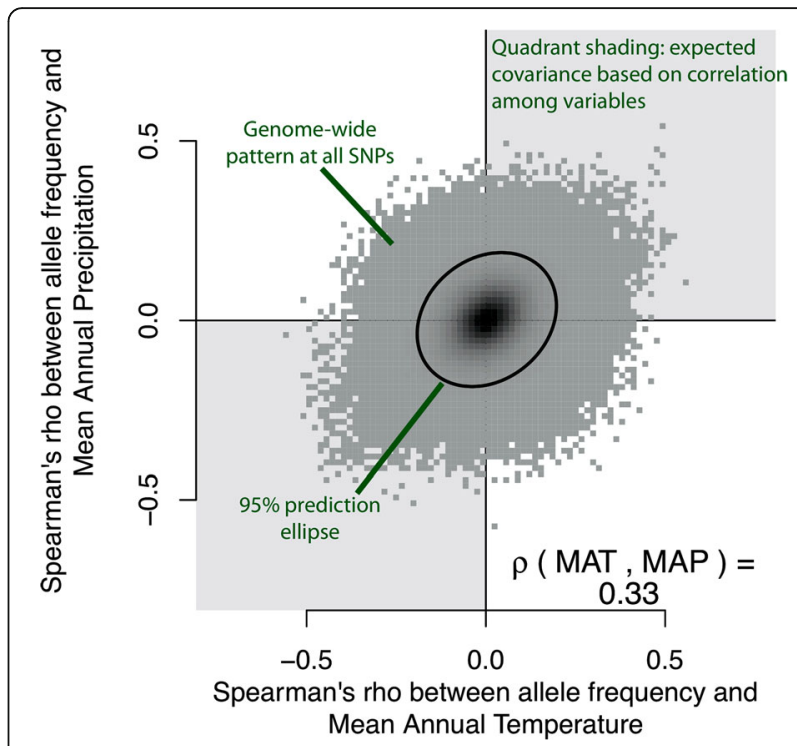

Fig. 4 Overview of galaxy biplots. The association between allele frequency and one variable is plotted against the association between allele frequency and a second variable. The Spearman's $\rho$ correlation between the two variables (mean annual temperature or MAT and mean annual precipitation or MAP in this example) is shown in the lower right corner. When the two variables are correlated, genome-wide covariance is expected to occur in the direction of their association (shown with quadrant shading in light gray). The observed genome-wide distribution of allelic effects is plotted in dark gray, and the 95\% prediction ellipse is plotted as a black line. Because derived alleles were coded as 1 and ancestral alleles were coded as 0 , the location of any particular SNP in bivariate space represents the type of environment that the derived allele is found in higher frequency, whereas the location of the ancestral allele would be a reflection through the origin (note only derived alleles are plotted)

the Freezing group showed associations with elevation but not latitude (compare height of blue points on $y$ axis of Fig. $5 \mathrm{c}-\mathrm{e}$ ). Conversely, the large co-association module in the Multi group (gene no. 1, dark green points) showed associations with latitude but not elevation, while the second co-association module in the Multi group (gene nos. 2-3, light green points) showed associations with both latitude and elevation (compare height of points on $y$ axis of Fig. $5 \mathrm{c}-\mathrm{e}$ ). Note how the structure correction polarized these patterns somewhat without changing interpretation, suggesting that the structurecorrected allelic associations become more extreme when their pattern of allele frequency contrasted the background population structure (compare left column of Fig. 5 to right column of Fig. 5).

Some modules were particularly defined by the fact that almost all the derived alleles changed frequency in the same direction (e.g., sweep-like signatures). For instance, for the co-association module in the Multi group defined by gene nos. 2-3, 14, of the 16 derived SNPs were found in higher frequencies at colder temperatures, 

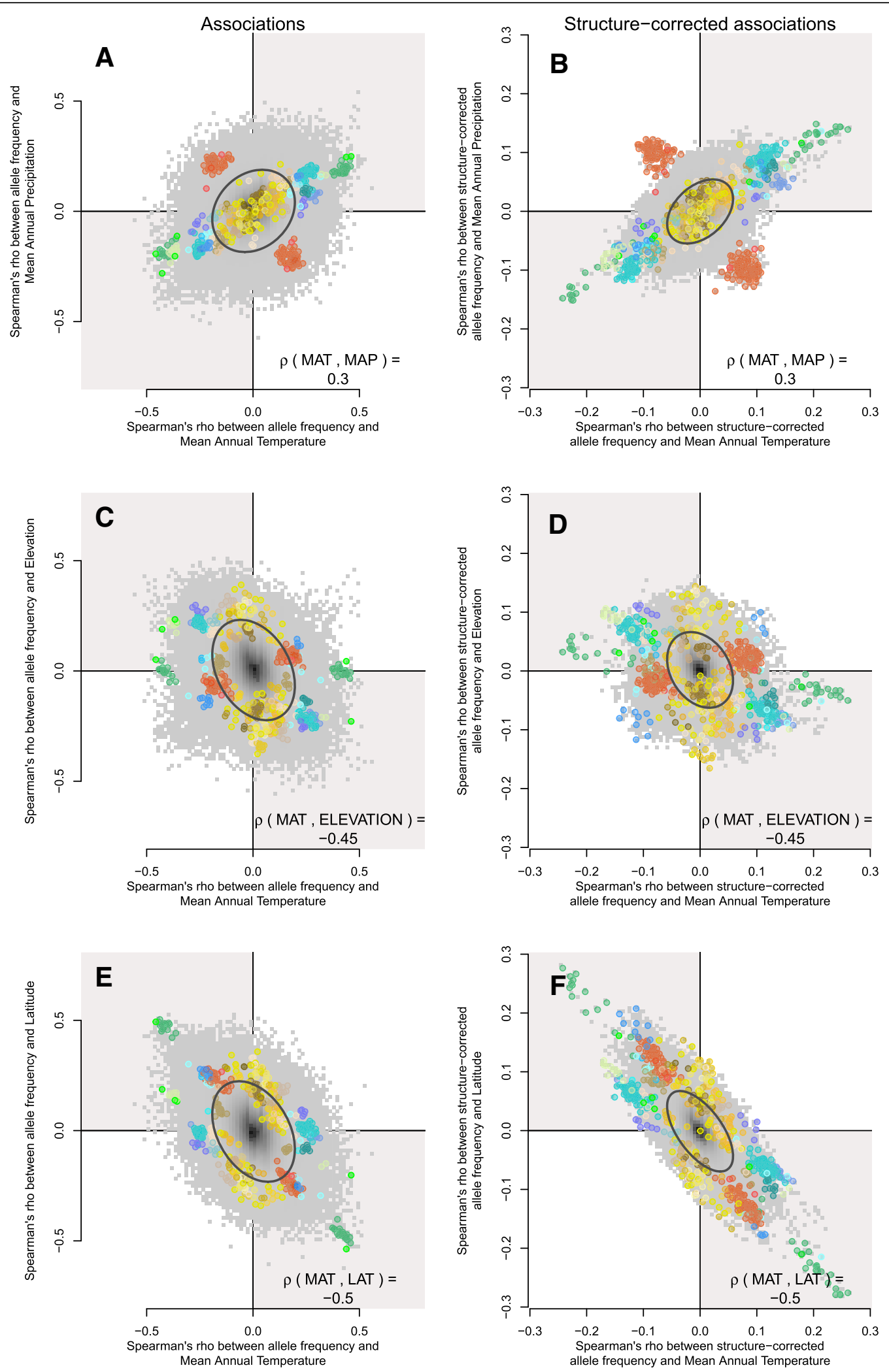

Fig. 5 (See legend on next page.) 
(See figure on previous page.)

Fig. 5 Galaxy biplots for different environmental variables for regular associations (left column) and structure-corrected associations (right column). Top candidate SNPs are highlighted against the genome-wide background. The correlation shown in the lower right corner represents Spearman's $\rho$ between the two environmental variables on the landscape. The internal color of each point corresponds to its co-association module (as shown in Fig. 2c-f). Top row: mean annual temperature (MAT) vs. mean annual precipitation (MAP), middle row: MAT and elevation, bottom row: MAT and latitude (LAT)

higher elevations, and higher latitudes. Contrast this with a group of SNPs from an co-association module in the Freezing group defined by gene no. 32, in which 14 of 15 derived SNPs were found in higher frequencies in warmer temperatures and lower elevations, but showed no associations with latitude. These may be candidates for genotypes that have risen in frequency to adapt to particular environmental conditions on the landscape.

Conversely, other modules showed different combinations of derived alleles that arose in frequency at opposite values of environmental variables. For instance, derived alleles in the Aridity co-association module were found in higher frequency in either warm, dry environments (88 of 155 SNPs) or in cold, moist environments (67 of 155 SNPs). Similarly, for the Multi co-association module defined by gene no. 1, derived alleles were found in higher frequency in either cold, dry environments (15 of 37 SNPs), or in warm, moist environments (22 of 37 SNPs). These may be candidates for genes acted on by antagonistic pleiotropy within a locus (Table 1), in which one genotype is selected for at one extreme of the environment and another genotype is selected for at the other extreme of the environment. Unfortunately, we were unable to fully characterize the relative abundance of sweep-like vs. antagonistically pleiotropic patterns across all top candidate genes due to (i) the low number of candidate SNPs for most genes, and (ii) for many SNPs, the derived allele could not be determined (because there was a SNP or missing data in the ancestral species).

We also visualized the patterns of allele frequency on the landscape for two representative SNPs, chosen because they had the highest number of connections in their co-association module (and were more likely to be true positives, see the Results section "Simulated datasets"). Geographic and climatic patterns are illustrated with maps for two such SNPs: (i) a SNP in the Multi co-association module with significant associations with latitude and mean annual temperature (Fig. 6a, gene no. 1 from Fig. 2) and (ii) a SNP in the Aridity co-association module with significant associations with annual heat:moisture index and latitude (Fig. 6b, gene no. 8 from Fig. 2). These maps illustrate the complex environments that may be selecting for particular combinations of genotypes despite potentially high gene flow in this widespread species.

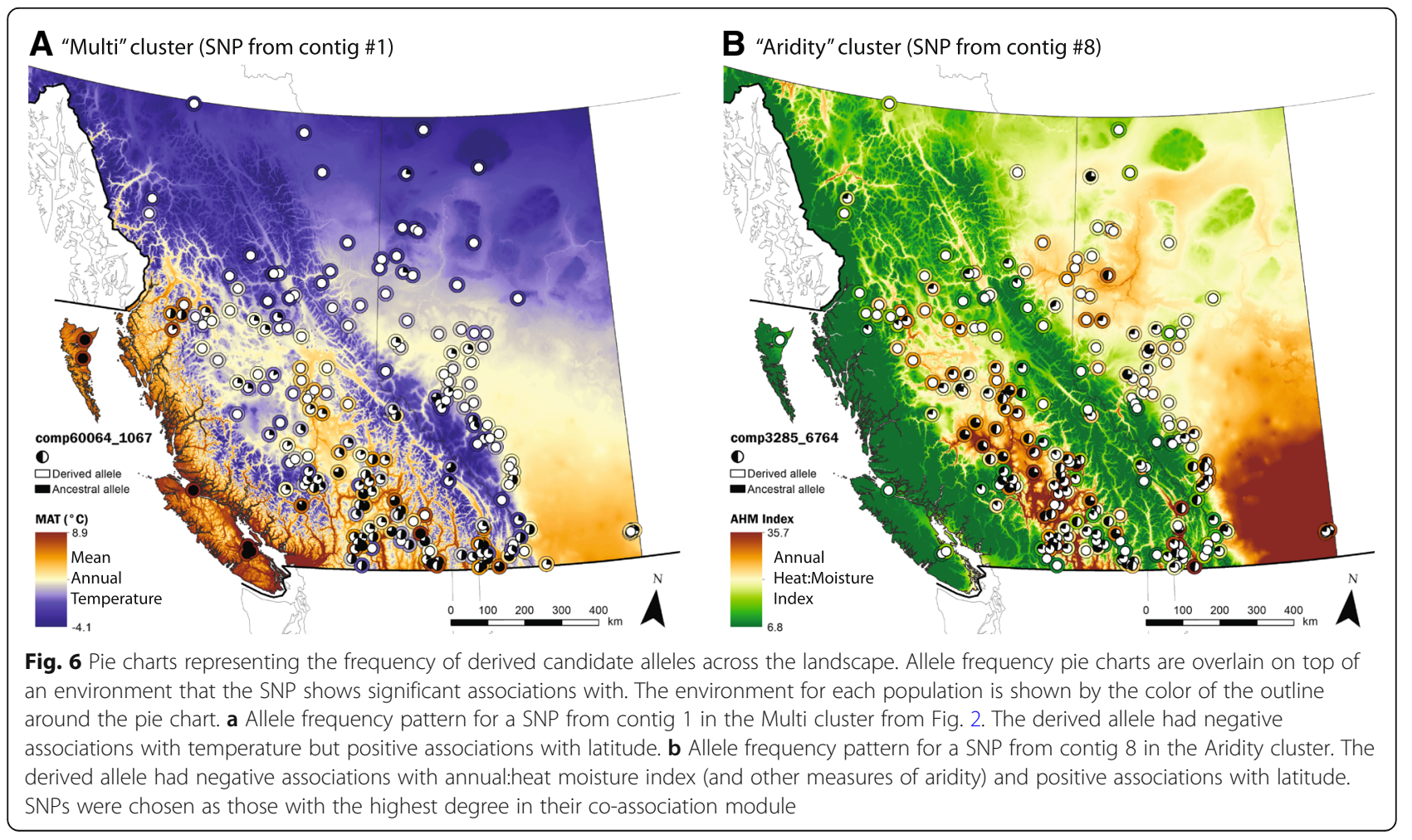




\section{Candidate gene annotations}

Although many of the candidate genes were not annotated, as is typical for conifers, the genes underlying adaptation to these environmental gradients had diverse putative functions. The top candidate SNPs were found in $3^{\prime}$ and $5^{\prime}$ untranslated regions and open reading frames in higher proportions than all exome SNPs (Additional file 1: Figure S11). A gene ontology (GO) analysis using previously assigned gene annotations [46, 55] found that a single molecular function, solute:cation antiporter activity, was over-represented across all top candidate genes (Additional file 2: Table S1). In the Aridity and Geography groups, annotated genes included sodium or potassium ion antiporters (one in Aridity, a KEA4 homolog, and two in Geography, NHX8 and SOS1 homologs), suggestive of a role in drought, salt or freezing tolerance [56]. Genes putatively involved in auxin biosynthesis were also identified in the Aridity (YUCCA 3) and Geography (Anthranilate synthase component) groups (Additional file 3: Table S2), suggestive of a role in plant growth. In the Freezing and Geography groups, several flowering time genes were identified [57] including a homolog of CONSTANS [58] in the Freezing group and a homolog of FY, which affects FCA mRNA processing, in the Geography group [58] (Additional file 3: Table S2). In addition, several putative drought/stress response genes were identified, such as DREB transcription factor [59] and an RCD1-like gene (Additional file 3: Table S2). RCD-1 is implicated in hormonal signaling and in the regulation of several stress-responsive genes in Arabidopsis thaliana [57]. In the Multi group, the only gene that was annotated functions in acclimation of photosynthesis to the environment in A. thaliana [60].

Of the 47 candidate genes identified by Yeaman et al. [46] as undergoing convergent evolution for adaptation to low temperatures in lodgepole pine and the interior spruce hybrid complex (Picea glauca, P. engelmannii, and their hybrids), 10 were retained with our stringent criteria for top candidates. All of these genes grouped into the Freezing and Geography groups (shown by an asterisk in Fig. 2g): the two groups that had many SNPs with significant associations with elevation. This is consistent with the pattern of local adaptation in the interior spruce hybrid zone, whereby Engelmann spruce is adapted to higher elevations and white spruce is adapted to lower elevations [61].

\section{Comparison of co-expression clusters to co-association modules}

To further explore if co-association modules have similar gene functions, we examined their gene expression patterns in response to climate treatments using previously published RNAseq data of 10,714 differentially expressed genes that formed eight distinct co-expression clusters [55]. Of the 108 top candidate genes, 48 (44\%) were also differentially expressed among treatments in response to factorial combinations of temperature (cold, mild, or hot), moisture (wet vs. dry), and/or day length (short vs. long day length). We found limited correspondence between co-association modules and co-expression clusters. Most of the top candidate genes that were differentially expressed mapped to two of the ten co-expression clusters previously characterized by [55] (Fig. 7, blue circles are the P2 co-expression cluster and green triangles are the P7 co-expression cluster previously described by [55]). Genes in the P2 co-expression cluster had functions associated with the regulation of transcription and their expression was strongly influenced by all treatments, while genes in the P7 co-expression cluster had functions relating to metabolism, photosynthesis, and response to stimulus [55]. Genes from the closely linked Aridity group mapped to four distinct co-expression clusters, contigs from the Freezing group mapped to three distinct co-expression clusters, and genes from the Geography group mapped to three distinct co-expression clusters.

We used a Fisher exact test to determine if any co-expression cluster was over-represented in any of the four major co-association groups shown in Fig. 2. We found that the Freezing group was over-represented in the P2 co-regulated gene expression cluster $(P<0.05)$ with seven $(58 \%)$ of the Freezing genes found within the P2 expression cluster, revealing coordinated expression in response to climatic conditions. Homologs of four of the seven genes were present in A. thaliana, and three of these genes were transcription factors involved in abiotic stress response (DREB transcription factor), flowering time (CONSTANS, pseudo-response regulator) or the circadian clock (pseudo-response regulator 9). No other significant over-representation of gene expression class was identified for the four association groups or for all adaptation candidate genes.

\section{Simulated datasets}

We used individual-based simulations to examine potential limitations of the co-association network analysis by comparing the connectedness of co-association networks arising from false positive neutral loci vs. a combination of false positive neutral loci and true positive loci that had experienced selection to an unmeasured environmental factor. Specifically, we used simulations with random sampling designs from three replicates across three demographic histories: (i) isolation by distance at equilibrium (IBD), (ii) non-equilibrium range expansion from a single refugium (1R), or from (iii) two refugia (2R). These landscape simulations were similar to lodgepole pine in the sense that they simulated large effective population sizes and resulted in similar $F_{S T}$ across the 


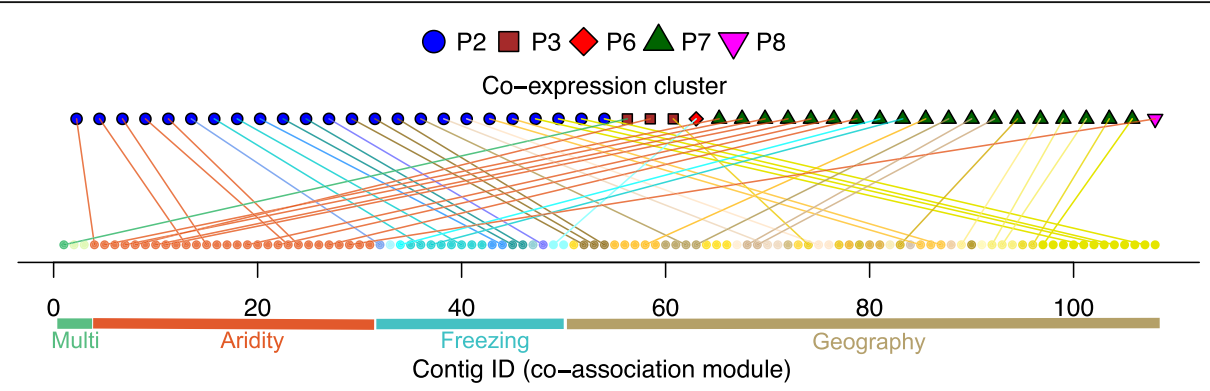

Fig. 7 Co-association modules mapped to co-expression clusters determined by climate treatments. Gene ID, color, and order shown on the bottom correspond to co-association modules plotted in Fig. 2. Co-expression clusters from [55] are shown at the top

landscape as that observed in pine $\left([62,63], F_{S T}\right.$ in simulations $\sim 0.05$, vs. $F_{S T}$ in pine $\left.\sim 0.016[46]\right)$. To explore how the allele frequencies that evolved in these simulations might yield spurious patterns under the co-association network analysis, we overlaid the 22 environmental variables used in the lodgepole pine dataset onto the landscape genomic simulations [62, 63]. To simulate selection to an unmeasured environmental factor, a small proportion of SNPs (1\%) were subjected to computer-generated spatially varying selection along a weak latitudinal cline $[62,63]$. We assumed that 22 environmental variables were measured, but not the "true" selective environment; our analysis thus represents the ability of co-association networks to correctly cluster selected loci even when the true selective environment was unmeasured, but a number of other environmental variables were measured (correlations between the selective environment and the other variables ranged from 0 to 0.2 ). Note that the simulations differ from the empirical data in at least two ways: (i) there is only one selective environment (so we can evaluate whether a single selective environment could result in multiple co-association modules in the data given the correlation structure of observed environments) and (ii) loci were unlinked.

The $P$ value and Bayes factor criteria for choosing top candidate SNPs in the empirical data produced no false positives with the simulated datasets (Additional file 1: Figure S12 right column), although using these criteria also reduced the proportion of true positives. Therefore, we used less stringent criteria to analyze the simulations so that we could also better understand patterns created by unlinked, false positive neutral loci (Additional file 1: Figure S12 left column).

We found that loci under selection by the same environmental factor generally formed a single tightly connected co-association module even though they were unlinked and that the degree of connectedness of selected loci was greater than among neutral loci (Fig. 8). Thus, a single co-association module typically resulted from adaptation to the single selective environment in the simulations. This occurred because the distance threshold used to define connections in the co-association modules was chosen as one that enriched for connections among selected loci with non-random associations in allele frequencies due to selection by a common environmental factor (Additional file 1: Figure S13).

The propensity of neutral loci to form tightly clustered co-association networks increased with the complexity of demographic history (compare Fig. 8 IBD in the left column to $2 \mathrm{R}$ in the right column). For example, the false positive neutral loci from the two-refugia (2R) model formed tightly connected networks, despite the fact that all simulated loci were unlinked. This occurred because of non-random associations in allele frequency due to a shared demographic history. In some cases, selected loci formed separate or semi-separate modules according to their strengths of selection, but the underlying patterns of association were the same (e.g., Figure 8a, Additional file 1: Figure S14).

\section{Discussion}

Co-association networks provide a valuable framework for interpreting the genetic architecture of local adaptation to the environment in lodgepole pine. Our most interesting result was the discovery of low recombination rates among genes putatively adapting to different and distinct aspects of climate, which was unexpected because selection is predicted to increase recombination between loci acted on by different sources of selection. If the loci we studied were true causal loci, then different sources of selection were strong enough to reduce LD among physically linked loci in the genome, resulting in modular effects of loci on fitness in the environment. While the top candidate SNPs from most genes had associations with only a single environmental factor, for some genes, we discovered evidence of environmental pleiotropy, i.e., candidate SNPs associated with multiple distinct aspects of climate. Within co-association modules, we observed a combination of local sweep-like signatures (in which derived alleles at a locus were all 


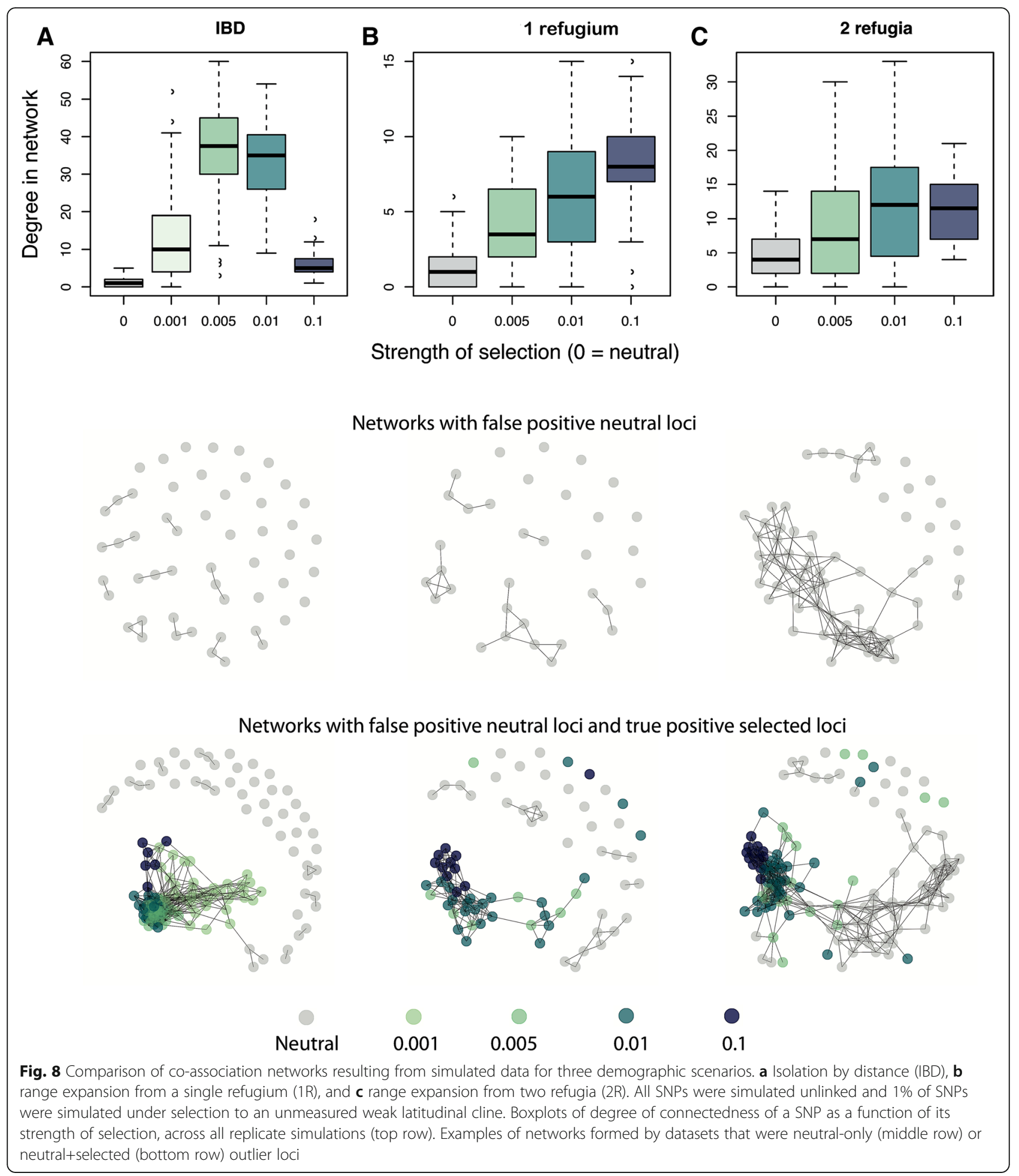

found in a particular climate, e.g., cold environments) and antagonistically pleiotropic patterns underlying adaptation to climate (in which some derived alleles at a locus were found at one environmental extreme and others found at the opposite extreme), although we could not evaluate the relative importance of these patterns. Finally, we observed that the modularity of candidate genes in their transcriptionally plastic responses to climate factors did not correspond to the modularity of these genes in their patterns of association with climate, as evidenced through comparing co-association networks with co-expression networks. These results give insight 
into evolutionary debates about the extent of modularity and pleiotropy in the evolution of genetic architecture [18-24].

\section{Genetic architecture of adaptation: pleiotropy and modularity}

Most of the top candidate genes in our analysis do not exhibit universal pleiotropy to distinct aspects of climate as defined by the expected pattern outlined in Fig. 1b. Our results are more consistent with the Hypothesis of Modular Pleiotropy [19], in which loci may have extensive effects within a distinct aspect of the environment (as defined by the variables that associate with each co-association module), but few pleiotropic effects among distinct aspects of the environment. These results are in line with theoretical predictions that modular architectures should be favored when there are many sources of selection in complex environments [26]. But note also that if many pleiotropic effects are weak, the stringent statistical thresholds used in our study to reduce false positives may also reduce the extent to which pleiotropy is inferred [20, 21]. Therefore in our study, any pleiotropic effects of genes on fitness detected in multiple aspects of climate are likely to be large effects, and we refrain to making any claims as to the extent of environmental pleiotropy across the entire genome.

The extent of pleiotropy within individual co-association modules is hard to quantify, as for any given module, we observed associations between genes and several environmental variables. Associations between a SNP and multiple environmental variables may or may not be interpreted as extensive environmental pleiotropic effects, depending on whether univariate environmental variables are considered distinct climatic factors or collectively represent a single multivariate optimum. In many cases, these patterns are certainly affected by correlations among the environmental variables themselves.

Our results also highlight conceptual issues with the definition of and interpretation of pleiotropic effects on distinct aspects of fitness from real data: namely, what constitutes a "distinct aspect" (be it among traits, components of fitness, or aspects of the environment)? In this study, we defined the selective environment through the perspective of those environmental variables we tested for associations with SNPs, using a threshold that produced reasonable results in simulation. But even with this definition, some co-association modules are more similar in their multivariate environmental "niche" than others. For instance, genes within the Geography group could be interpreted to have extensive pleiotropic effects if the patterns of associations of each individual module were taken to be "distinct," or they may be considered to have less extensive pleiotropic effects if their patterns of associations were too similar to be considered "distinct." While the framework we present here is a step toward understanding and visualizing this hierarchical nature of "distinct aspects" of environmental factors, a more formal framework is needed to quantify the distinctness of pleiotropic effects.

\section{Genetic architecture of adaptation: linkage}

We also observed physical linkage among genes that were associated with very distinct aspects of climate. This was somewhat unexpected from a theoretical perspective: while selection pressures due to genome organization may be weak, if anything, selection would be expected to disfavor linkage and increase recombination between genes adapting to selection pressures with different spatial patterns of variation [34-36]. Interestingly, while the recombination rate analysis suggests that these loci are sometimes located relatively close together on a single chromosome, this does not seem to be sufficient physical linkage to also cause a noticeable increase in LD. In other words, it is possible that the amount of physical linkage sometimes observed between genes in different co-association modules is not strong enough to constrain adaptation to these differing gradients. Genetic maps and reference genomes are not yet well developed for the large genomes of conifers; improved genetic maps or assembled genomes will be required to explore these questions in greater depth. If this finding is robust and not compromised by false positives, physical linkage among genes adapting to different climatic factors could either facilitate or hinder a rapid evolutionary response as the multivariate environment changes $[4,5]$.

Within co-association modules, we observed varying patterns of physical linkage among genes. The Aridity group, in particular, consisted of several tightly linked genes that may have arisen for a number of different reasons. Clusters of physically linked genes such as this may act as a single large-effect QTL [64] and may have evolved due to competition among alleles or genomic rearrangements ([30], although these are rare in conifers), increased establishment probability due to linked adaptive alleles [4], or divergence within inversions [32]. Alternatively, if the Aridity region was one of low recombination, a single causal variant could create the appearance of linked selection [65], a widespread false positive signal may have arisen due to genomic variation such as background selection and increased drift [6668], or a widespread false signal may have arisen due to a demographic process such as allele surfing $[69,70]$.

\section{Genetic architecture of adaptation: modularity of transcriptional plasticity vs. fitness}

We also compared co-expression networks to co-association networks. Genes that showed similar responses in expression 
in lodgepole pine seedlings in response to experimental climatic treatments form a co-expression network. Since co-expression networks have been successful at identifying genes that respond the same way to environmental stimuli [71], it might be reasonable to expect that if these genes were adapting to climate they would also show similar patterns of associations with climate variables. However, differential expression analyses only identify genes with plastic transcriptional responses to climate. Plasticity is not a prerequisite for adaptation and may be an alternative strategy to adaptation. This is illustrated by our result that only half of our top candidate contigs for adaptation to climate were differentially expressed in response to climate conditions.

Interestingly, loci located within the same co-association module (groups of loci that are putatively favored or linked to loci putatively favored by natural selection) could be found in different co-expression clusters. For example, we observed that loci from the tightly linked Aridity module had many distinct expression patterns in response to climate treatments. Conversely, candidate genes that were associated with different aspects of the multivariate environment (because they were located in different co-association modules) could nonetheless be co-expressed in response to specific conditions. These observations support the speculation that the developmental/functional modularity of plasticity may not correspond to the modularity of the genotype to fitness map; however, the power of the analysis could be low due to stringent statistical cutoffs and these patterns warrant further investigation.

\section{Physiological adaptation of lodgepole pine to climate}

It is challenging to disentangle the physiological effects and importance of freezing versus drought in the local adaptation of conifers to climate. We found distinct groups of candidate genes along an axis of warm/wet to cold/dry (co-association modules in the Freezing and Multi groups), and another distinct group along an axis of cold/wet to warm/dry (the Aridity co-association module). Selection by drought conditions in winter may occur through extensive physiological remodeling that allows cells to survive intercellular freezing by desiccating protoplasts-but also results in drought stress at the cellular level [55]. Another type of winter drought injury in lodgepole pine-red belt syndrome-is caused by warm, often windy events in winter, when foliage desiccates but the ground is too cold for roots to be able to supply water above ground [72]. This may contrast with drought selection in summer, when available soil water is lowest and aridity highest. The physiological and cellular mechanisms of drought and freezing response have similarities but also potentially important differences that could be responsible for the patterns we have observed.

Our results provide a framework for developing hypotheses that will help to disentangle selective environments and provide genotypes for assisted gene flow in reforestation [73]. While climate change is expected to increase average temperatures across this region, some areas are experiencing more precipitation than historic levels and others experiencing less [74]. Tree mortality rates are increasing across North America due to increased drought and vapor pressure deficit for tree species including lodgepole pine, and associated increased vulnerability to damaging insects, but growth rates are also increasing with warming temperatures and increased carbon dioxide [75, 76]. Hot, dry valleys in southern $\mathrm{BC}$ are projected to have novel climates emerge that have no existing analogues in North America [77]. The considerable standing adaptive variation we observe here involving many genes could facilitate adaptation to new temperature and moisture regimes, or could hinder adaptation if novel climates are at odds with the physical linkage among alleles adapted to different climate stressors.

\section{Limitations of associations with principal components}

For these data, testing associations of genes with PC-based climate variables would have led to a very limited interpretation of the environmental drivers of selection because the PC ordination is not biologically informed as to what factors are driving divergent selection [37]. First, many putative candidates in the Freezing and Geography groups would have been missed. Second, strong associations between the Multi SNPs and environmental variables that did not load strongly onto PC1, such as latitude, would have also been missed. Finally, many Aridity SNPs were significantly associated in PC3, which was a PC axis that had strong correlations with environmental variables that the Aridity SNPs did not have any significant associations with. This occurred because no single environmental variable loaded strongly onto PC3 (the maximum loading of any single variable was 0.38 ) and many variables had moderate loadings, such that no single variable explained the majority of the variance (the maximum variance explained by any one variable was $15 \%$ ). Thus, associations with higher PC axes become increasingly difficult to interpret when the axis itself explains less variance of the multivariate environment and the environmental factors loading onto that axis explain similar amounts of variance in that axis. While principal components will capture the environmental factors that covary the most, this may have nothing to do with the combinations that drive divergent selection and local adaptation. This needlessly adds a layer of complexity to an analysis that may not reveal anything 
biologically important. In contrast, co-association networks highlight those combinations of environments that are biologically important for those genes likely involved in local adaptation.

\section{Benefits and caveats of co-association networks}

Co-association networks provide an intuitive and visual framework for understanding patterns of associations of genes and SNPs across many potentially correlated environmental variables. By parsing loci into different groups based on their associations with multiple variables, this framework offers a more informative approach than grouping loci according to their outlier status based on associations with single environmental variables. While in this study we have used them to infer groups of loci that adapt to distinct aspects of the multivariate environment, co-association networks could be widely applied to a variety of situations, including genotype-phenotype associations. They offer the benefit of jointly identifying modules of loci and the groups of environmental variables that the modules are associated with. While the field may still have some disagreement about how modularity and pleiotropy should be defined, measured, and interpreted [19-21, 23, 24], co-association networks at least provide a quantitative framework to define and visualize modularity.

Co-association networks differ from the application of bipartite network theory for estimating the degree of classical pleiotropic effects of genes on traits [3]. Bipartite networks are two-level networks where the genes form one type of nodes and the traits form the second type of nodes, then a connection is drawn from a gene to a trait if there is a significant association [3]. The degree of pleiotropy of a locus is then inferred by the number of traits that a gene is connected to. With the bipartite network approach, trait nodes are defined by those traits measured, and not necessarily the multivariate effects from the perspective of the gene (e.g., a gene that affects organism size will have effects on height, weight, and several other variables, and if all these traits are analyzed, this gene would be inferred to have large pleiotropic effects). Even if highly correlated traits are removed, simulations have shown that even mild correlations in mutational effects can bias estimates of pleiotropy from bipartite networks $[20,21]$. The advantage of co-association networks is their ability to identify combinations of variables (be they traits or environments) that associate with genetic (or SNP) modules. Correlated variables that measure essentially the same environment or phenotype will simply cluster together in a module, which can facilitate interpretation. On the other hand, correlated variables that measure different aspects of the environment or phenotype may cluster into different modules (as we observed in this study).
The observed combinations of associations can then be used to develop and test hypotheses as to whether the genotype-environment combination represents a single multivariate environment that the gene is adapting to (in the case of allele associations with environment or fitness) or a single multivariate trait that the gene affects (in the case of allele associations with phenotypes). This approach may complement other machine-learning approaches based on multivariate associations with environments [78], which is a promising avenue for future research.

While co-association networks hold promise for elucidating the modularity and pleiotropy of the genotype-phenotype-fitness map, some caveats should be noted. First, correlations among variables will make it difficult to infer the exact conditions that select for or the exact traits that associate with particular allelic combinations. Results from this framework can make it easier, however, to generate hypotheses that can be tested with future experiments. Second, the analysis of simulated data shows that investigators should consider demographic history and choose candidates with caution for data analysis to exclude false positives, as we have attempted here. Co-association networks can arise among unlinked neutral loci by chance, and it is almost certain that some proportion of the "top candidate SNPs" in this study are false positives due to linkage with causal SNPs or due to demographic history. The simulated data also showed, however, that causal SNPs tend to have a higher degree of connection in their co-association network than neutral loci, and this might help to prioritize SNPs for follow up experiments, SNP arrays, and genome editing. Third, it may be difficult to draw conclusions about the level of modularity of the genetic architecture. The number of modules may be sensitive to the statistical thresholds used to identify top candidate SNPs $[20,21]$ as well as the distance threshold used to identify modules. With our data, the number of co-associations modules and the number of SNPs per module were not very sensitive to increasing this threshold by 0.05 , but our results were sensitive to decreasing the threshold 0.05 (a stricter threshold resulted in smaller modules of SNPs with extremely similar associations, and a large number of "modules" comprised of a single SNP unconnected to other SNPs-even SNPs in the same gene) (results not shown). While inferred modules composed of a single SNP could be interpreted as unique, our simulations also show that neutral loci are more likely to be unconnected in co-association networks. Many alleles of small effect may be just below statistical detection thresholds, and whether or not these alleles are included could profoundly change inference as to the extent of pleiotropy [20,21]. This presents a conundrum common to most population genomic approaches for detecting selection, because lowering statistical thresholds will 
almost certainly increase the number of false positives, while only using very stringent statistical thresholds may decrease the probability of observing pleiotropy if many pleiotropic effects are weak [20]. Thus, while co-association networks are useful for identifying SNP modules associated with correlated variables, further work is necessary to expand this framework to quantitatively measure pleiotropic effects in genomes.

\section{Conclusions}

In this study, we discovered physical linkage among loci putatively adapting to different aspects of the climate. These results give rare insight into both the ecological pressures that favor the evolution of modules by natural selection [19] and into the organization of genetic architecture itself. As climate changes, the evolutionary response will be determined by the extent of physical linkage among these loci, in combination with the strength of selection and phenotypic optima across environmental gradients, the scale and pattern of environmental variation, and the details of migration and demographic fluctuations across the landscape. While theory has made strides to provide a framework for predicting the genetic architecture of local adaptation under divergence with gene flow to a single environment [4, $30,31,79-83]$, as well as the evolution of correlated traits under different directions and/or strengths of selection when those traits have a common genetic basis $[35,36]$, how genetic architectures evolve on complex heterogeneous landscapes has not been clearly elucidated. Furthermore, it has been difficult to test theory because the field still lacks frameworks for evaluating empirical observations of adaptation in many dimensions. Here, we have attempted to develop an initial framework for understanding adaptation to several complex environments with different spatial patterns, which may also be useful for understanding the genetic basis of multivariate phenotypes from genome-wide association studies. This framework lays the foundation for future studies to examine modularity across the genotype-phenotype-fitness continuum.

\section{Methods}

\section{Sampling and climate}

This study uses the same dataset analyzed by Yeaman et al. [46], but with a different focus as explained in the introduction. Briefly, we obtained seeds from 281 sampling locations of lodgepole pine (Pinus contorta) from reforestation collections for natural populations, and these locations were selected to represent the full range of climatic and ecological conditions within the species range in British Columbia and Alberta based on ecosystem delineations. Seeds were grown in a common garden and 2-4 individuals were sampled from each sampling location. The environment for each sampling location was characterized by estimating climate normals for 1961-1990 from geographic coordinates using the software package ClimateWNA [84]. The program extracts and downscales the moderate spatial resolution generated by PRISM [85] to scale-free and calculates many climate variables for specific locations based on latitude, longitude, and elevation. The downscaling is achieved through a combination of bilinear interpolation and dynamic local elevational adjustment. We obtained 19 climatic and three geographical variables (latitude, longitude, and elevation). Geographic variables may correlate with some unmeasured environmental variables that present selective pressure to populations (e.g., latitude correlates with day length). Many of these variables were correlated with each other on the landscape (Fig. 2a).

\section{Sequencing, bioinformatics, and annotation}

The methods for this section are identical to those reported in [46]. Briefly, DNA from frozen needle tissue was purified using a Macherey-Nagel Nucleospin 96 Plant II Core kit automated on an Eppendorf EpMotion 5075 liquid handling platform. One microgram of DNA from each individual tree was made into a barcoded library with a $350 \mathrm{bp}$ insert size using the BioO NEXTflex Pre-Capture Combo kit. Six individually barcoded libraries were pooled together in equal amounts before sequence capture. The capture was performed using custom Nimblegen SeqCap probes ([46] for more details, see [47]) and the resulting captured fragments were amplified using the protocol and reagents from the NEXTflex kit. All sample preparation steps followed the recommended protocols provided. After capture, each pool of six libraries was combined with another completed capture pool and the 12 individually barcoded samples were then sequenced, 100-bp paired-end, on one lane of an Illumina HiSeq 2500 (at the McGill University and Genome Quebec Innovation Centre).

Sequenced reads were filtered and aligned to the loblolly pine genome [86] using bwa mem [87] and variants were called using GATK Unified Genotyper [88], with steps included for removal of PCR duplicates, realignment around indels, and base quality score recalibration $[46,88]$. SNP calls were filtered to eliminate variants that did not meet the following cutoffs: quality score $>=20$, map quality score $>=45$, FisherStrand score $<=33$, HaplotypeScore $<$ $=7$, MQRankSumTest < = 12.5, ReadPosRankSum > 8, and allele balance $<2.2$, minor allele frequency $>5 \%$, and genotyped successfully in $>10 \%$ of individuals. Ancestral alleles were coded as a 0 and derived alleles coded as a 1 for data analysis. 
We used the annotations developed for pine in [46]. Briefly, we performed a BLASTX search against the TAIR 10 protein database and identified the top blast hit for each transcript contig ( $e$ value cut-off was $10^{-6}$ ). We also performed a BLASTX against the nr (non-redundant) database screened for green plants and used Blast2GO [89] to assign GO terms and enzyme codes ([46] for details, see [55]). We also assigned GO terms to each contig based on the GO A thaliana mappings and removed redundant GO terms. To identify if genes with particular molecular function and biological processes were over-represented in top candidate genes, we performed a GO enrichment analysis using topGO [90]. All GO terms associated with at least two candidate genes were analyzed for significant over-representation within each group and in all candidate genes (FDR 5\%).

\section{Top candidate SNPs}

First, top candidate genes were obtained from [46]. For this study, genes with unusually strong signatures of association from multiple association tests (uncorrected genotype-phenotype and genotype-environment correlations, for details see [46]) were identified as those with more outlier SNPs than expected by chance with a probability of $P<10^{-9}$, which is a very restrictive cutoff (note that due to non-independence among SNPs in the same contig, this $P$ value is an index, and not an exact probability). Thus, the subsequent analysis is limited to loci that we have the highest confidence are associated with adaptation as evidenced by a large number of significant SNPs (not necessarily the loci with the largest effect sizes).

For this study, we identified top candidate SNPs within the set of top candidate genes. These "top candidate SNPs" had allele-environment associations with (i) $P$ values lower than the Bonferroni cutoff for the uncorrected Spearman's $\rho\left(\sim 10^{-8}=0.05 /\right.$ (number of SNPs times the number of environmental variables) and (ii) $\log _{10}(\mathrm{BF})>2$ for the structure-corrected Spearman's $\rho$ (Bayenv2, for details see below). The resulting set of candidate SNPs rejects the null hypothesis of no association with the environment with high confidence. In subsequent analyses, we interpret the results both before and after correction for population structure, to ensure that structure correction does not change our overall conclusions. Note that because candidate SNPs are limited to the top candidate genes in order to reduce false positives in the analysis, these restrictive cutoffs may miss many true positives.

For uncorrected associations between allele frequencies and environments, we calculated the non-parametric rank correlation Spearman's $\rho$ between allele frequency for each SNP and each environmental variable. For structure-corrected associations between allele frequencies and environments, we used the program Bayenv2 [39]. Bayenv2 is implemented in two steps. In the first step, the variance-covariance matrix is calculated from allelic data. As detailed in [46], a set of non-coding SNPs was used to calculate the variance-covariance matrix from the final run of the MCMC after 100,000 iterations, with the final matrix averaged over three MCMC runs. In the second step, the variance-covariance matrix is used to control for evolutionary history in the calculation of test statistics for each SNP. For each SNP, Bayenv2 outputs a Bayes factor (a value that measures the strength of evidence in favor of a linear relationship between allele frequencies and the environment after population structure is controlled for) and Spearman's $\rho$ (the non-parametric correlation between allele frequencies and environment variables after population structure is controlled for). Previous authors have found that the stability of Bayes factors is sensitive to the number of iterations in the MCMC [91]. We ran three replicate chains of the MCMC with 50,000 iterations, which we found produced stable results. Bayes factors and structure-corrected Spearman's $\rho$ were averaged over these three replicate chains, and these values were used for analysis.

\section{Co-association networks}

We first organized the associations into a matrix with SNPs in columns, environments in rows, and the specific SNP-environment association in each cell. These data were used to calculate pairwise Euclidean distances between SNPs based on their associations, and this distance matrix was used to cluster SNP loci with Ward's hierarchical clustering using the hclust function in the $\mathrm{R}$ package stats [92]. As described in the results, this resulted in four main groups in the data. For each of these main groups, we used undirected graph networks to visualize submodules of SNPs. Nodes (SNPs) were connected by edges if they had a pairwise Euclidean distance less than 0.1 from the distance matrix described above. We found that the results were not very sensitive to this distance threshold. Co-association networks were visualized using the igraph package in R v 1.0.1 [93].

\section{Linkage disequilibrium}

Linkage disequilibrium was calculated among pairwise combinations of SNPs within genes. Mean values of Pearson's correlation coefficient squared $\left(r^{2}\right)$ were estimated across all SNPs annotated to each pair of individual genes, excluding SNPs genotyped in fewer than 250 individuals (to minimize the contribution of small sample sizes to the calculation of gene-level means).

\section{Recombination rates}

An Affymetrix SNP array was used to genotype 95 full-sib offspring from a single cross of two parents. 
Individuals with genotype posterior probabilities of > 0.001 were filtered out. This array yielded data for 13,544 SNPs with mapping-informative genotypes. We used the package "onemap" in $\mathrm{R}$ with default settings to estimate recombination rates among pairs of loci, retaining all estimates with LOD scores $>3$ [94]. This dataset contained 2760 pairs of SNPs that were found together on the same genomic contig, separated by a maximum distance of $13-\mathrm{k}$ base pairs. Of these 7,617,600 possible pairs, 521 were found to have unrealistically high inferred rates of recombination $(r>0.001)$, and are likely errors. These errors probably occurred as a result of the combined effect of undetected errors in genotype calling, unresolved paralogy in the reference genome that complicates mapping, and differences between the reference loblolly genome that was used for SNP design and the lodgepole pine genomes. As a result, recombination rates that were low $(r<0.001)$ were expected to be relatively accurate, but we do not draw any inferences about high recombination estimates among loci.

\section{Associations with principal components of environments}

To compare inference from co-association networks to another multivariate approach, we conducted a principal components analysis of environments using the function prcomp () in R. Then, we used Bayenv2 to test associations with $\mathrm{PC}$ axes as described above and used $\mathrm{BF}>2$ as a criterion for the significance of a SNP on a PC axis. Note that this criterion is less conservative than that used to identify candidate SNPs for the network analysis (because it did not require the additional criterion of a significant Bonferroni-corrected $P$ value), so it should result in greater overlap between PC candidate SNPs and top candidate SNPs based on univariate associations.

\section{Enrichment of co-expressed genes}

The co-expression data used in this study was previously published by [55]. To determine if adaptation cluster members had similar gene functions, we examined their gene expression patterns in response to seven growth chamber climate treatments using previously published RNAseq data [55]. Expression data was collected on 44 seedlings from a single sampling location, raised under common conditions, and then exposed to growth chamber environments that varied in their temperature, moisture, and photoperiod regimes. We used Fisher's exact test to determine if genes with a significant climate treatment effect were over-represented in each of the four major groups and across all adaptation candidates relative to the other sequenced and expressed genes. In addition, Yeaman et al. [55] used weighted gene co-expression network analysis (WGCNA) to identify eight clusters of co-regulated genes among the seven climate treatments. We used a Fisher's exact test to determine if these previously identified expression clusters were over-represented in the any of the four major groups relative to the other sequenced and expressed genes.

\section{Galaxy biplots}

To give insight into how the species has evolved to inhabit multivariate environments relative to the ancestral state, we visualized the magnitude and direction of associations between the derived allele frequency and environmental variables. Allelic correlations with any pair of environmental variables can be visualized by plotting the value of the non-parametric rank correlation Spearman's $\rho$ of the focal allele with variable 1 against the value with variable 2 . Spearman's $\rho$ can be calculated with or without correction for population structure. Note also that the specific location of any particular allele in a galaxy biplot depends on the way alleles are coded. SNP data were coded as 0,1 , or 2 copies of the loblolly reference allele. If the reference allele has positive Spearman's $\rho$ with temperature and precipitation, then the alternate allele has a negative Spearman's $\rho$ with temperature and precipitation. For this reason, the alternate allele at a SNP should be interpreted as a reflection through the origin (such that quadrants 1 and 3 are symmetrical and quadrants 2 and 4 are symmetrical if the reference allele is randomly chosen).

A prediction ellipse was used to visualize the genome-wide pattern of covariance in allelic effects on a galaxy biplot. For two variables, the $2 \times 2$ variance-covariance matrix of $\operatorname{Cov}\left(\rho\left(f, E_{1}\right), \rho\left(f, E_{2}\right)\right)$, where $f$ is the allele frequency and $E_{x}$ is the environmental variable, has a geometric interpretation that can be used to visualize covariance in allelic effects with ellipses. The covariance matrix defines both the spread (variance) and the orientation (covariance) of the ellipse, while the expected values or averages of each variable $\left(E\left[E_{1}\right]\right.$ and $E\left[E_{2}\right]$ ) represent the centroid or location of the ellipse in multivariate space. The geometry of the two-dimensional $(1-\alpha) \times 100 \%$ prediction ellipse on the multivariate normal distribution can then be approximated by

$$
l_{j}=\sqrt{\lambda_{j} \mathrm{X}_{d f=2, \alpha}}
$$

where $l_{j}$ represents the lengths of the major $(j=1)$ and minor $(j=2)$ axes on the ellipse, respectively, $\lambda_{j}$ represents the eigenvalues of the covariance matrix, and $\chi^{2} \mathrm{df}=$ $2, \alpha$ represents the value of the $\chi^{2}$ distribution for the desired $\alpha$ value [95-97]. In the results, we plot the 95\% prediction ellipse $(\alpha=0.05)$ corresponding to the volume within which $95 \%$ of points should fall assuming the data is multivariate normal, using the function ellipsoidPoints() in the $\mathrm{R}$ package cluster [98]. This approach will 
work when there is a large number of unlinked SNPs in the set being visualized; if used on a candidate set with a large number of linked SNPs and/or a small candidate set with non-random assignment of alleles (i.e., allele assigned according to a reference), the assumptions of this visualization approach will be violated.

\section{Visualization of allele frequencies on the landscape}

ESRI ArcGIS v10.2.2 was used to visualize candidate SNP frequencies across the landscape. Representative SNPs having the most edges within each sub-network were chosen and plotted against climatic variables representative of those co-association modules. Mean allele frequencies were calculated for each sampled population and plotted. Climate data and $1-\mathrm{km}$ resolution rasters were obtained using ClimateWNA v5.40 [84] and shaded with color gradients scaled to the range of climates across the sampling locations. The climates for each sampling location were also plotted, as some sampling locations were at especially high or low elevations relative to their surrounding landscapes. For clarity, only sampling locations containing at least two sampled individuals were plotted.

\section{Simulations}

The simulations used in this study are identical to a subset of those previously published by $[62,63]$. Briefly, the simulator uses forward-in-time recurrence equations to model the evolution of independent haploid SNPs on a quasi-continuous square landscape. We modeled three demographic histories that resulted in the same overall neutral $F_{S T}$ for each demography, but demographic history determined the distribution of $F_{S T}$ 's around that mean. Isolation by distance (IBD) had the lowest variance, followed by demographic expansion from a single refuge (1R), and demographic expansion from two refugia $2 \mathrm{R}$ had the highest variance. The landscape size was $360 \times 360$ demes, and migration was determined by a discretized version of a Gaussian dispersal kernel. Carrying capacity per deme differed slightly for each scenario to give the same overall neutral $F_{S T}=0.05$. IBD was run until equilibrium at 10,000 generations, but $1 \mathrm{R}$ and $2 \mathrm{R}$ were only run for 1000 generations in order to mimic the expansion of lodgepole pine since the last glacial maximum [99]. All selected loci adapted to a computer-generated landscape with a weak north-south cline and spatial heterogeneity at smaller spatial scales with varying strengths of selection from weak $(s=0.001)$ to strong $(s=0.1)$, see $[62,63]$ for more details.

The simulations were then expanded in the following way: for each of the 22 environmental variables for lodgepole pine populations, we used interpolation to estimate the value of the variable at the simulated locations. This strategy preserved the correlation structure among the 22 environmental variables. For each of the 22 variables, we calculated the uncorrected rank correlation (Spearman's $\rho$ ) between allele frequency and environment. The 23rd computer-generated environment was not included in analysis, as it was meant to represent the hypothetical situation that there is a single unmeasured (and unknown) environmental variable that is the driver of selection. The 23rd environment was correlated from 0 to 0.2 with the other 22 variables.

We compared two thresholds for determining which loci were retained for co-association network analysis, keeping loci with either: (i) a $P$ value lower than the Bonferroni correction $(0.05 /$ (no. environments * no. simulated loci)) and (ii) a log-10 Bayes factor (BF) $>2$ (for at least one of the environmental variables). Using both criteria is more stringent and both were used in the lodgepole pine analysis. In the simulations, however, we found that using both criteria resulted in no false positives in the outlier list (see the "Results" section); therefore we used only the first of these two criteria so that we could understand how false positives may affect interpretation of the co-association network analysis. For a given set of outliers (e.g., only false positives or false positives and true positives), hierarchical clustering and undirected graph networks were built in the same manner as described for the lodgepole pine data.

\section{Additional files}

Additional file 1: Supplementary Figures. Fourteen supplementary figures associated with the manuscript. (PDF 12466 kb)

Additional file 2: Table S1. Results from $\mathrm{GO}$ analysis for all top candidate genes and for each group. (XLSX $9 \mathrm{~kb}$ )

Additional file 3: Table S2. Top candidate genes and their annotations, (XLSX $72 \mathrm{~kb})$

\section{Abbreviations}

LD: Linkage disequilibrium; PC: Principal components; SNP: Single-nucleotide polymorphism

\section{Acknowledgements}

We thank Sebastian E. Ramos-Onsins, Tanja Pyhäjärvi, an anonymous reviewer, and PCl Evol Biol for comments that greatly improved this manuscript. Mike Whitlock provided valuable advice and feedback on various aspects of the research. We thank Jeremy Yoder for organizing the SNP chip data used for calculating the recombination rates. Pia Smets, Connor Fitzpatrick, and Sarah Markert assembled and grew genetic materials, and Kristin Nurkowski prepared sequence capture libraries. Tongli Wang and Andreas Hamann selected populations based on climatic distribution of species. Seeds were kindly donated by 63 forest companies and agencies in Alberta and British Columbia (listed at http://adaptree.forestry.ubc.ca/seed-contributors/).

\section{Funding}

KEL was supported by a grant from the National Science Foundation (502483). This research was part of the AdapTree project, led by SNA and by Genome Canada (LSARP2010_161REF), Genome BC, Genome Alberta, Alberta Innovates BioSolutions, the Forest Genetics Council of British Columbia, the British Columbia Ministry of Forests, Lands and Natural Resource Operations (BCMFLNRO), Virginia Polytechnic University, and the University of British Columbia. 


\section{Availability of data and materials}

The datasets and analysis code supporting the conclusions of this article are published on Dryad https://doi.org/10.5061/dryad.r67hd7t [100]

\section{Authors' contributions}

KEL conceived of the analysis, conducted analyses, and lead writing of the manuscript. KH and SY did the bioinformatics and various specific analyses. JD created the allele frequency landscape plots. SA led the AdapTree project. All authors contributed to the writing of the manuscript. All authors read and approved the final manuscript.

\section{Ethics approval and consent to participate}

Not applicable.

\section{Consent for publication}

Not applicable.

\section{Competing interests}

The authors declare that they have no competing interests.

\section{Publisher's Note}

Springer Nature remains neutral with regard to jurisdictional claims in published maps and institutional affiliations.

\section{Author details}

Department of Marine and Environmental Sciences, Northeastern Marine Science Center, 430 Nahant Rd, Nahant, MA 01908, USA. ²Department of Biological Sciences, University of Calgary, Calgary, AB T2N1N4, Canada. ${ }^{3}$ Department of Forest and Conservation Sciences, Faculty of Forestry, Vancouver, BC V6T 1Z4, Canada. ${ }^{4}$ School of Biological Sciences, Monash University, Wellington Rd, Clayton, Melbourne, VIC 3800, Australia.

\section{Received: 25 January 2018 Accepted: 18 September 2018}

\section{Published online: 05 October 2018}

\section{References}

1. Hansen TF. The evolution of genetic architecture. Annu Rev Ecol Evol Syst. 2006;37:123-57

2. Orr HA. Adaptation and the cost of complexity. Evolution. 2000:54:13-20.

3. Wang Z, Liao B-Y, Zhang J. Genomic patterns of pleiotropy and the evolution of complexity. Proc Natl Acad Sci U S A. 2010;107:18034-9.

4. Aeschbacher S, Bürger R. The effect of linkage on establishment and survival of locally beneficial mutations. Genetics. 2014;197:317-36.

5. Reeve J, Ortiz-Barrientos D, Engelstädter J. The evolution of recombination rates in finite populations during ecological speciation. Proc Biol Sci. 2016; 283. https://doi.org/10.1098/rspb.2016.1243.

6. Barton NH. Genetic linkage and natural selection. Philos Trans R Soc Lond B Biol Sci. 2010;365:2559-69.

7. Wagner GP, Zhang J. The pleiotropic structure of the genotype-phenotype map: the evolvability of complex organisms. Nat Rev Genet. 2011:12:204-13.

8. Paaby AB, Rockman MV. The many faces of pleiotropy. Trends Genet. 2013; 29:66-73.

9. Savolainen $\mathrm{O}$, Lascoux M, Merilä J. Ecological genomics of local adaptation. Nat Rev Genet. 2013;14:807-20.

10. Slatkin M. Gene flow and selection in a cline. Genetics. 1973:75:733-56.

11. Slatkin M. Spatial patterns in the distributions of polygenic characters. J Theor Biol. 1978:70:213-28.

12. Barton NH. Clines in polygenic traits. Genet Res. 1999;74:223-36.

13. Felsenstein J. The theoretical population genetics of variable selection and migration. Annu Rev Genet. 1976;10:253-80.

14. Haldane JBS. The theory of a cline. J Genet. 1948;48:277-84.

15. Haldane JBS. A mathematical theory of natural and artificial selection (Part VI, Isolation). Math Proc Cambridge Philos Soc. 1930;26:220.

16. Rellstab C, Gugerli F, Eckert AJ, Hancock AM, Holderegger R. A practical guide to environmental association analysis in landscape genomics. Mol Ecol. 2015;24:4348-70.

17. Hancock AM, Brachi B, Faure N, Horton MW, Jarymowycz LB, Sperone FG, et al. Adaptation to climate across the Arabidopsis thaliana genome. Science. 2011;334:83-6.

18. Boyle EA, Li YI, Pritchard JK. An expanded view of complex traits: from polygenic to omnigenic. Cell. 2017;169:1177-86.
19. Wagner GP, Pavlicev M, Cheverud JM. The road to modularity. Nat Rev Genet. 2007:8:921-31.

20. Hill WG, Zhang X-S. Assessing pleiotropy and its evolutionary consequences: pleiotropy is not necessarily limited, nor need it hinder the evolution of complexity. Nat Rev Genet. 2012. https://doi.org/10.1038/nrg2949-c1.

21. Hill WG, Zhang X-S. On the pleiotropic structure of the genotypephenotype map and the evolvability of complex organisms. Genetics. 2012; 190:1131-7.

22. Rockman MV. The QTN program and the alleles that matter for evolution: all that's gold does not glitter. Evolution. 2012;66:1-17.

23. Paaby AB, Rockman MV. Pleiotropy: what do you mean? Reply to Zhang and Wagner. Trends Genet. 2013;29:384.

24. Wagner GP, Zhang J. Universal pleiotropy is not a valid null hypothesis: reply to Hill and Zhang. Nat Rev Genet. 2012;13:296.

25. Wagner GP. Homologues, natural kinds and the evolution of modularity. Am Zool. 1996;36:36-43.

26. Le Nagard H, Chao L, Tenaillon O. The emergence of complexity and restricted pleiotropy in adapting networks. BMC Evol Biol. 2011;11:326.

27. Griswold CK. Pleiotropic mutation, modularity and evolvability. Evol Dev. 2006;8:81-93.

28. Le Corre V, Kremer A. Genetic variability at neutral markers, quantitative trait loci and trait in a subdivided population under selection. Genetics. 2003; 164:1205-19.

29. Hill WG, Robertson A. The effect of linkage on limits to artificial selection. Genet Res. 1966;8:269-94.

30. Yeaman S. Genomic rearrangements and the evolution of clusters of locally adaptive loci. Proc Natl Acad Sci U S A. 2013;110:E1743-51.

31. Yeaman S, Aeschbacher S, Bürger R. The evolution of genomic islands by increased establishment probability of linked alleles. Mol Ecol. 2016; 25:2542-58

32. Kirkpatrick M. Chromosome inversions, local adaptation and speciation. Genetics. 2006:173:419-34.

33. Schwander T, Libbrecht R, Keller L. Supergenes and complex phenotypes. Curr Biol. 2014;24:R288-94

34. Lenormand T, Otto SP. The evolution of recombination in a heterogeneous environment. Genetics. 2000;156:423-38.

35. Guillaume F. Migration-induced phenotypic divergence: the migrationselection balance of correlated traits. Evolution. 2011;65:1723-38.

36. Chebib J, Guillaume F. What affects the predictability of evolutionary constraints using a G-matrix? The relative effects of modular pleiotropy and mutational correlation. Evolution. 2017. https://doi.org/10.1111/evo.13320.

37. Houle D, Mezey J, Galpern P. Interpretation of the results of common principal components analyses. Evolution. 2002;56:433-40.

38. Frichot $\mathrm{E}$, Schoville SD, Bouchard G, François O. Testing for associations between loci and environmental gradients using latent factor mixed models. Mol Biol Evol. 2013;30:1687-99.

39. Günther T, Coop G. Robust identification of local adaptation from allele frequencies. Genetics. 2013;195:205-20.

40. Gautier M. Genome-wide scan for adaptive divergence and association with population-specific covariates. Genetics. 2015;201:1555-79.

41. Lasky JR, Des Marais DL, McKay JK, Richards JH, Juenger TE, Keitt TH. Characterizing genomic variation of Arabidopsis thaliana: the roles of geography and climate. Mol Ecol. 2012;21:5512-29.

42. Benestan L, Quinn BK, Maaroufi H, Laporte M, Clark FK, Greenwood SJ, et al. Seascape genomics provides evidence for thermal adaptation and currentmediated population structure in American lobster (Homarus americanus). Mol Ecol. 2016:25:5073-92

43. Hedrick PW. Genetic polymorphism in heterogeneous environments: a decade later. Annu Rev Ecol Syst. 1986;17:535-66.

44. Hedrick PW, Ginevan ME, Ewing EP. Genetic polymorphism in heterogeneous environments. Annu Rev Ecol Syst. 1976;7:1-32.

45. Barton NH. Multilocus clines. Evolution. 1983:37:454-71.

46. Yeaman S, Hodgins KA, Lotterhos KE, Suren H, Nadeau S, Degner JC, et al. Convergent local adaptation to climate in distantly related conifers. Science. 2016;353:1431-3.

47. Suren $H$, Hodgins KA, Yeaman S, Nurkowski KA, Smets $P$, Rieseberg LH, et al. Exome capture from the spruce and pine giga-genomes. Mol Ecol Resour. 2016:16:1136-46.

48. Hodgins KA, Yeaman S, Nurkowski KA, Rieseberg LH, Aitken SN. Expression divergence Is correlated with sequence evolution but not positive selection in conifers. Mol Biol Evol. 2016;33:1502-16. 
49. Eckert AJ, Bower AD, González-Martínez SC, Wegrzyn JL, Coop G, Neale DB. Back to nature: ecological genomics of loblolly pine (Pinus taeda, Pinaceae). Mol Ecol. 2010;19:3789-805.

50. Eckert AJ, van Heerwaarden J, Wegrzyn JL, Nelson CD, Ross-Ibarra J, González-Martínez SC, et al. Patterns of population structure and environmental associations to aridity across the range of loblolly pine (Pinus taeda L., Pinaceae). Genetics. 2010;185:969-82.

51. Alberto FJ, Aitken SN, Alía R, González-Martínez SC, Hänninen H, Kremer A et al. Potential for evolutionary responses to climate change-evidence from tree populations. Glob Chang Biol. 2013;19:1645-61.

52. Howe GT, Aitken SN, Neale DB, Jermstad KD, Wheeler NC, Chen THH. From genotype to phenotype: unraveling the complexities of cold adaptation in forest trees. Can J Bot. 2003:81:1247-66.

53. Liepe KJ, Hamann A, Smets P, Fitzpatrick CR, Aitken SN. Adaptation of lodgepole pine and interior spruce to climate: implications for reforestation in a warming world. Evol Appl. 2016;9:409-19.

54. Illingworth K. Study of lodgepole pine genotype-environment interaction in B.C. In: Proceedings International Union of Forestry Research Organizations (IUFRO) Joint Meeting of Working parties: Douglas-fir provenances, Lodgepole Pine Provenances, Sitka Spruce Provenances and Abies Provenances. Vancouver, British Columbia; 1978. p. 151-158.

55. Yeaman S, Hodgins KA, Suren H, Nurkowski KA, Rieseberg LH, Holliday JA, et al. Conservation and divergence of gene expression plasticity following c. 140 million years of evolution in lodgepole pine (Pinus contorta) and interior spruce (Picea glaucaxPicea engelmannii). New Phytol. 2014;203:578-91.

56. Blumwald E, Aharon GS, Apse MP. Sodium transport in plant cells. Biochimica et Biophysica Acta (BBA) - Biomembranes. 2000;1465:140-51.

57. Ahlfors $R$, Lång $S$, Overmyer $K$, Jaspers $P$, Brosché $M$, Tauriainen $A$, et al. Arabidopsis RADICAL-INDUCED CELL DEATH1 belongs to the WWE proteinprotein interaction domain protein family and modulates abscisic acid, ethylene, and methyl jasmonate responses. Plant Cell. 2004;16:1925-37.

58. Amasino RM, Michaels SD. The timing of flowering. Plant Physiol. 2010;154: 516-20.

59. Singh D, Laxmi A. Transcriptional regulation of drought response: a tortuous network of transcriptional factors. Front Plant Sci. 2015;6:895.

60. Walters RG, Shephard F, Rogers JJM, Rolfe SA, Horton P. Identification of mutants of Arabidopsis defective in acclimation of photosynthesis to the light environment. Plant Physiol. 2003;131:472-81.

61. De La Torre A, Ingvarsson PK, Aitken SN. Genetic architecture and genomic patterns of gene flow between hybridizing species of Picea. Heredity. 2015; 115:153-64

62. Lotterhos KE, Whitlock MC. Evaluation of demographic history and neutral parameterization on the performance of $F_{\text {ST }}$ outlier tests. Mol Ecol. 2014;23: 2178-92.

63. Lotterhos KE, Whitlock MC. The relative power of genome scans to detect local adaptation depends on sampling design and statistical method. Mol Ecol. 2015:24:1031-46.

64. Christians JK, Senger LK. Fine mapping dissects pleiotropic growth quantitative trait locus into linked loci. Mamm Genome. 2007:18:240-5.

65. Charlesworth B, Nordborg M, Charlesworth D. The effects of local selection, balanced polymorphism and background selection on equilibrium patterns of genetic diversity in subdivided populations. Genet Res. 1997;70:155-74.

66. Charlesworth B. The effects of deleterious mutations on evolution at linked sites. Genetics. 2012;190:5-22.

67. Charlesworth B, Morgan MT, Charlesworth D. The effect of deleterious mutations on neutral molecular variation. Genetics. 1993;134:1289-303.

68. Hoban S, Kelley JL, Lotterhos KE, Antolin MF, Bradburd G, Lowry DB, et al. Finding the genomic basis of local adaptation: pitfalls, practical solutions, and future directions. Am Nat. 2016;188:379-97.

69. Klopfstein $S$, Currat M, Excoffier $L$. The fate of mutations surfing on the wave of a range expansion. Mol Biol Evol. 2006;23:482-90.

70. Hofer T, Ray N, Wegmann D, Excoffier L. Large allele frequency differences between human continental groups are more likely to have occurred by drift during range expansions than by selection. Ann Hum Genet. 2009;73:95-108.

71. Zhang B, Horvath S. A general framework for weighted gene co-expression network analysis. Stat Appl Genet Mol Biol. 2005:4:Article 17.

72. Bella IE, Navratil S. Growth losses from winter drying (red belt damage) in lodgepole pine stands on the east slopes of the Rockies in Alberta. Can J For Res. 1987;17:1289-92.

73. Aitken SN, Whitlock MC. Assisted gene flow to facilitate local adaptation to climate change. Annu Rev Ecol Evol Syst. 2013;44:367-88.
74. Mbogga MS, Hamann A, Wang T. Historical and projected climate data for natural resource management in western Canada. Agric For Meteorol. 2009; 149:881-90.

75. Hember RA, Kurz WA, Coops NC. Relationships between individual-tree mortality and water-balance variables indicate positive trends in water stress-induced tree mortality across North America. Glob Chang Biol. 2017; 23:1691-710.

76. Hember RA, Kurz WA, Coops NC. Increasing net ecosystem biomass production of Canada's boreal and temperate forests despite decline in dry climates. Global Biogeochem Cycles. 2017;31:2016GB005459.

77. Mahony CR, Cannon AJ, Wang T, Aitken SN. A closer look at novel climates: new methods and insights at continental to landscape scales. Glob Chang Biol. 2017. https://doi.org/10.1111/gcb.13645.

78. Fitzpatrick MC, Keller SR. Ecological genomics meets community-level modelling of biodiversity: mapping the genomic landscape of current and future environmental adaptation. Ecol Lett. 2015;18:1-16.

79. Yeaman S, Whitlock MC. The genetic architecture of adaptation under migration-selection balance. Evolution. 2011;65:1897-911.

80. Kremer A, Le Corre V. Decoupling of differentiation between traits and their underlying genes in response to divergent selection. Heredity. 2012;108: 375-85.

81. Le Corre V, Kremer A. The genetic differentiation at quantitative trait loci under local adaptation. Mol Ecol. 2012;21:1548-66.

82. Flaxman SM, Feder JL, Nosil P. Genetic hitchhiking and the dynamic buildup of genomic divergence during speciation with gene flow. Evolution. 2013; 67:2577-91.

83. Bürger $\mathrm{R}$, Akerman $\mathrm{A}$. The effects of linkage and gene flow on local adaptation: A two-locus continent-island model. Theor Popul Biol. 2011:80:272-88.

84. Wang T, Hamann A, Spittlehouse DL, Murdock TQ. ClimateWNA—highresolution spatial climate data for western North America. J Appl Meteorol Climatol. 2012:51:16-29.

85. Daly C, Halbleib M, Smith Jl, Gibson WP, Doggett MK, Taylor GH, et al. Physiographically sensitive mapping of climatological temperature and precipitation across the conterminous United States. Int J Climatol. 2008;28: 2031-64.

86. Neale DB, Wegrzyn JL, Stevens KA, Zimin AV, Puiu D, Crepeau MW, et al. Decoding the massive genome of loblolly pine using haploid DNA and novel assembly strategies. Genome Biol. 2014;15:R59.

87. Li H, Durbin R. Fast and accurate short read alignment with BurrowsWheeler transform. Bioinformatics. 2009:25:1754-60.

88. DePristo MA, Banks E, Poplin R, Garimella KV, Maguire JR, Hartl C, et al. A framework for variation discovery and genotyping using next-generation DNA sequencing data. Nat Genet. 2011;43:491-8.

89. Conesa A, Götz S. Blast2GO: a comprehensive suite for functional analysis in plant genomics. Int J Plant Genomics. 2008;2008:619832.

90. Alexa A, Rahnenführer J. Gene set enrichment analysis with topGO. 2009. https://bioconductor.riken.jp/packages/3.2/bioc/vignettes/topGO/inst/doc/ topGO.pdf. Accessed 1 Jan 2017.

91. Blair LM, Granka JM, Feldman MW. On the stability of the Bayenv method in assessing human SNP-environment associations. Hum Genomics. 2014:8:1.

92. Müllner D. fastcluster: fast hierarchical, agglomerative clustering routines for RandPython. J Stat Softw. 2013;53. https://doi.org/10.18637/jss.v053.i09.

93. Csardi G, Nepusz T. The igraph software package for complex network research. InterJournal Complex Syst. 2006;1695:1-9.

94. Margarido GRA, Souza AP, Garcia AAF. OneMap: software for genetic mapping in outcrossing species. Hereditas. 2007;144:78-9.

95. Pison G, Struyf A, Rousseeuw PJ. Displaying a clustering with CLUSPLOT. Comput Stat Data Anal. 1999;30:381-92.

96. Kaufman L, Rousseeuw PJ. Finding groups in data: an introduction to cluster analysis. New Jersey: Wiley Series in Probability and Statistics; 2009.

97. Titterington DM. Algorithms for computing D-optimal design on finite design spaces, Proceedings of the 1976 Conference on Information Science and Systems; 1976. p. 213-6.

98. Maechler M, Rousseeuw P, Struyf A, Hubert M, Hornik K. cluster: cluster analysis basics and extensions. 2018.

99. Hewitt G. The genetic legacy of the quaternary ice ages. Nature. 2000;405: 907-13.

100. Lotterhos KE, Yeaman S, Degner J, Aitken S, Hodgins KA. Data from: modularity of genes involved in local adaptation to climate despite physical linkage. https://doi.org/10.5061/dryad.r67hd7t. 